\title{
Obstacle Avoidance in a Dynamic Environment: A Collision Cone Approach
}

\author{
Animesh Chakravarthy and Debasish Ghose
}

\begin{abstract}
A novel collision cone approach is proposed as an aid to collision detection and avoidance between irregularly shaped moving objects with unknown trajectories. It is shown that the collision cone can be effectively used to determine whether collision between a robot and an obstacle (both moving in a dynamic environment) is imminent. No restrictions are placed on the shapes of either the robot or the obstacle, i.e., they can both be of any arbitrary shape. The collision cone concept is developed in a phased manner starting from existing analytical results-available in aerospace literature-that enable prediction of collision between two moving point objects. These results are extended to predict collision between a point and a circular object, between a point and an irregularly shaped object, between two circular objects, and finally between two irregularly shaped objects. Using the collision cone approach, several strategies that the robot can follow in order to avoid collision, are presented. A discussion on how the shapes of the robot and obstacles can be approximated in order to reduce computational burden is also presented. A number of examples are given to illustrate both collision prediction and avoidance strategies of the robot.
\end{abstract}

Index Terms - Collision cone, dynamic environments, obstacle avoidance, path planning.

\section{INTRODUCTION}

$\mathbf{O}$ BSTACLE avoidance is a fundamental requirement in motion planning of a mobile robot. Several papers addressing this issue have appeared in robotics literature [6], [8], [13], [23], [24]. Motion planning can be categorized [6] as static (when the obstacles are stationary in the environment) or dynamic (when the obstacles are capable of movement and may even change shape and size). The environment could be completely known (when the trajectory of the obstacles is known a priori) or partially known (when obstacle trajectory is unknown or information about it is incomplete). This classification is not universal and an alternative classification is available in [8]. To date, a major research effort in this area has been applied to analyze and solve the problem of motion planning in a completely known environment with largely static and, to some extent, moving obstacles [23]. Their primary goal was to determine a collision-free path from a starting point to a goal point while optimizing some performance criterion. Configuration space approach, Voronoi diagrams, retraction methods, potential functions, visibility graphs, accessibility graphs, tangent graphs, etc. ([6], [8],

Manuscript received August 27, 1996; revised December 7, 1997.

A. Chakravarthy is with the Flight Mechanics and Simulation Division, Aeronautical Development Agency, Bangalore 560 017, India.

D. Ghose is with the Department of Aerospace Engineering, Indian Institute of Science, Bangalore 560 012, India (e-mail: dghose@aero.iisc.ernet.in).

Publisher Item Identifier S 1083-4427(98)04346-X.
[16]) are some of the techniques which have been reasonably successful in achieving this goal. While these approaches are justifiable for a completely known environment, a partially known dynamic environment-which is a more realistic framework in situations where obstacle motion cannot be predicted-requires a different approach. In fact, dynamic motion planning is more difficult than static motion planning even when complete information about the environment is available. This is shown by several available complexity results for motion planning [21].

Recent advances in robotics technology has made possible the development of autonomous and semiautonomous robotic systems for land, air, and underwater operations. These robots use sophisticated onboard sensors to perceive their environment and use this information to plan and execute tasks [2], [3], [22], [23], [29]. Their primary use is in uncertain environments characterized by the presence of moving obstacles with unpredictable trajectories. Motion planning of robots in uncertain and unpredictable environments has attracted the attention of robotics researchers only recently [1], [7], [9]-[12], [19], [26], [25], [28].

In this paper, we present a novel approach called the collision cone approach which is ideally suited for automated guided vehicles or autonomous mobile robots. The method is new in the sense that it uses concepts which have their roots in aerospace literature rather than in robotics. The only relevant paper in the robotics literature that uses a similar concept is that by Tychonievich et al. [27].

The specific problem considered in this paper is that of a mobile robot avoiding one or more moving obstacles with unknown trajectories, based on sensor information collected by the robot. The robot and the obstacles are both assumed to move only by translation in a two-dimensional (2-D) space. Unlike previous literature, no assumptions are made on the shape of the robot or obstacles (i.e., they need not be polygons-convex, or otherwise). They can be of any arbitrary shape but with the constraint that each is a single rigid body without relative motion between points on the body. Thus, this approach is more suitable for the problem of obstacle avoidance of an automated guided vehicle or a mobile robot in a workspace consisting of moving obstacles, rather than for motion planning of robotic manipulators.

This paper is motivated by the conviction that collision avoidance and collision achievement are, in principle, two aspects of the same problem. In the robotics literature, the problem of collision avoidance has attracted a considerable amount of attention. On the other hand, collision achievement 


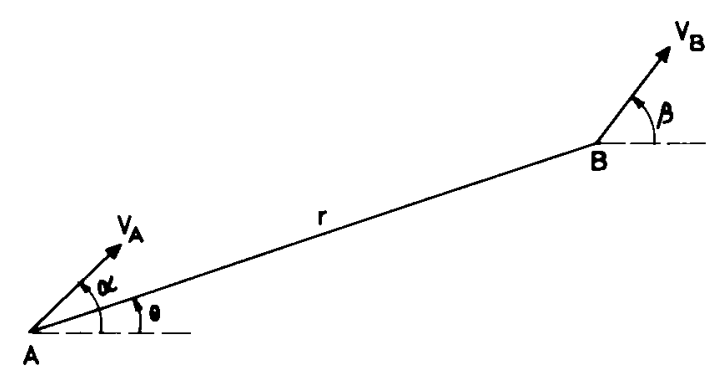

Fig. 1. Collision geometry between two point objects.

(or interception) has been of primary concern mainly in the aerospace guidance literature [14], [30]. For example, some of the problems addressed in the guidance literature involve the guidance of a missile to intercept an aircraft, guidance of a torpedo to intercept a ship, etc. The problem of achieving collision or interception is a long-standing area of research in the aerospace literature and has evolved into a reasonably complete theory, especially in the missile guidance literature, over the past five decades. Though the problem of collision avoidance in the robotics literature is of a more recent vintage, it has also witnessed intense research activity in recent years. However, to date there has been almost no cross-fertilization of ideas between the two areas. The main reason for this is the apparent dichotomy between them in terms of their objectives, operating environments, vehicle dynamics, sensor systems, performance capabilities, etc. With the present day technological advances (especially in sensor systems) and the stringent performance requirements on intelligent robotic vehicles [3], it is felt that many of the advances in the area of aerospace guidance theory has considerable relevance in robotics too.

Our purpose is to show that the fundamental concepts used for achieving intercept between bodies in motion can also be used to formulate strategies for collision avoidance. In doing so we extend these fundamental concepts to situations relevant to the collision avoidance problem in robotics, and derive several results that are new not only in robotics but also in aerospace guidance.

The literature on interception problems has mainly focussed upon conditions of collision between two moving point objects based on their instantaneous velocities. In the context of collision avoidance between robots it is not sufficient to consider an object as a point mass. Its physical shape and size, and its position and orientation in space, are of prime importance. We first extend the existing theory for predicting collision between point objects to obtain more general conditions to predict collision between objects of arbitrary shapes and sizes. Based on these results the collision cone is presented as a fundamental concept used for the purpose of collision avoidance. We restrict our study to the 2-D planar case in this paper.

This paper is organized as follows. In Section II, we review some results well-known in the aerospace guidance literature and illustrate some basic concepts through simple examples which also show how the collision cone can be obtained for a simple initial geometry between a point robot and a

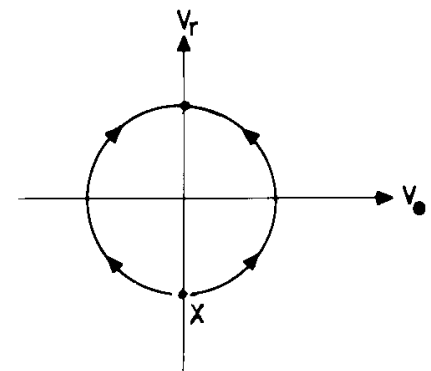

Fig. 2. A typical $\left(V_{\theta}, V_{r}\right)$ trajectory.

circular object. In Section III, we formalize the concept of the collision cone and present analytical results to obtain the exact collision cone between a point and a circular object, between a point and an irregularly shaped object, between two circular objects, and finally, between two irregularly shaped objects. In Section IV, we discuss some simple strategies for collision avoidance based on the collision cone concept. In Section V, we examine the possibility of simplifying the computation of the collision cone by approximating irregularly shaped objects as a collection of circular objects. We conclude the paper in Section VI with a general discussion on the applicability of the collision cone approach.

\section{Collision Between Point Objects}

The engagement geometry for an interception problem is given in Fig. 1. Here, $A$ and $B$ are two point objects, moving at constant velocities $V_{A}$ and $V_{B}$, respectively. The behavior of the line-of-sight (LOS) is characterized by the following kinematic equations:

$$
\begin{aligned}
& V_{r}=\dot{r}=V_{B} \cos (\beta-\theta)-V_{A} \cos (\alpha-\theta) \\
& V_{\theta}=r \dot{\theta}=V_{B} \sin (\beta-\theta)-V_{A} \sin (\alpha-\theta)
\end{aligned}
$$

where $V_{r}$ and $V_{\theta}$ are the relative velocity components (with respect to $A$ ) along, and perpendicular to, the LOS, respectively. Since we consider only the instantaneous velocities of the point objects, $\alpha$ and $\beta$ are assumed to be constants. Differentiating (1) and (2), we get

$$
\begin{aligned}
& \dot{V}_{r}=\dot{\theta} V_{B} \sin (\beta-\theta)-\dot{\theta} V_{A} \sin (\alpha-\theta)=\dot{\theta} V_{\theta} \\
& \dot{V}_{\theta}=-\dot{\theta} V_{B} \cos (\beta-\theta)+\dot{\theta} V_{A} \cos (\alpha-\theta)=-\dot{\theta} V_{r} .
\end{aligned}
$$

Dividing (3) by (4) and cross-multiplying, we get

$$
\dot{V}_{r} V_{r}+\dot{V}_{\theta} V_{\theta}=0
$$

which, on integration, yields the following relation:

$$
V_{r}^{2}+V_{\theta}^{2}=V_{r 0}^{2}+V_{\theta 0}^{2}
$$

where $V_{r 0}$ and $V_{\theta 0}$ are the relative velocity components at some initial time $\left(t=t_{0}\right)$. Equation (6) shows that the trajectory on the $\left(V_{\theta}, V_{r}\right)$ plane is a circle with center at the origin and radius equal to the initial relative velocity between $A$ and $B$. This also implies that the relative velocity is a constant with respect to time. Also, from (3), (4), and (6), it is evident that the trajectories in the $\left(V_{\theta}, V_{r}\right)$ plane have a time history shown in Fig. 2. Next, we state the following well-known results ([14], [15], [20], [30]). 
Lemma 1: The set of points satisfying the condition $V_{\theta}=0$ (for example, point $X$ in Fig. 2) are stationary points on the $\left(V_{\theta}, V_{r}\right)$ plane.

Proof: $V_{\theta}(=r \dot{\theta})=0$ implies that $\dot{\theta}=0$. Substituting this in (3) and (4) yields $\dot{V}_{r}=0$ and $\dot{V}_{\theta}=0$.

Lemma 2: If two point objects are moving with constant velocities (i.e., constant speed and direction), then $V_{\theta 0}=0$ and $V_{r 0}<0$, together form a necessary and sufficient condition for collision.

Proof: From Lemma 1, $V_{\theta 0}=0$ and $V_{r 0}<0$ implies that $V_{\theta}=0$ and $V_{r}<0$ for all future time. But $V_{\theta}=0$ implies that the LOS does not rotate in space and $V_{r}(=\dot{r})<0$ implies that distance between the two objects reduces until collision occurs, thus proving sufficiency.

To prove necessity, let the two objects collide at time $t=t_{f}$. In the time preceding $t_{f}$, the distance between the objects was obviously decreasing, thus implying that $V_{r}\left(t_{f}^{-}\right)<0$. If the positions of the objects are projected back to any time $t_{1}<t_{f}$, the resulting figure formed is a triangle with the LOS at $t_{1}$ as one side and the collision point as the opposite vertex so that successive lines of sight at times $t>t_{1}$ are parallel to each other. Thus, $V_{\theta}\left(t_{f}^{-}\right)=0$. From Lemma 1 , we can see that $V_{\theta}\left(t_{f}^{-}\right)=0 ; V_{r}\left(t_{f}^{-}\right)<0$ implies that $V_{\theta 0}=0 ; V_{r 0}<0$, thus proving necessity.

Example 1: Consider an initial geometry between a point object $O$ and a circle $\mathcal{F}$, with $r_{0}=10 ; \theta_{0}=45^{\circ} ; \beta=$ $60^{\circ} ; V_{O}=2 ; V_{\mathcal{F}}=1.5$ and $R=3$. Let $C$ be a point on the circle $\mathcal{F}$, at an angle $\phi=10^{\circ}$ with reference to $O P$. Then, by using (1) and (2), and the conditions indicated in Lemma 2 , it can be shown that $O$ is on a collision course with point $C$ if $\alpha=58.75^{\circ}$. See Fig. 3(a).

If we now consider all the points on the circle $\mathcal{F}$; then, by using (1) and (2) (and Lemma 2) for each such point, we get a corresponding value of $\alpha$ that causes $O$ to collide with that point. The collection of all the values of $\alpha$ defines a cone such that if $V_{O}$ lies in this cone then $O$ will eventually collide with $\mathcal{F}$. We refer to this cone as the collision cone. In this example, it is found that the collision cone is defined by $\alpha \epsilon\left[51.28^{\circ}, 60.61^{\circ}\right]$. See Fig. 3(b).

Example 2: Consider another initial geometry between $O$ and $\mathcal{F}$, with $r_{0}=10 ; \theta_{0}=45^{\circ} ; \beta=215^{\circ} ; V_{O}=2 ; V_{\mathcal{F}}=2.5$ and $R=3$. Again, $C$ is a point on the circle $\mathcal{F}$ at $\phi=10^{\circ}$. Now, $O$ will collide with $C$ if $\alpha=85.31^{\circ}$ or $\alpha=209.68^{\circ}$. The two values of $\alpha$ correspond to collision occurring at different points in space and at different instants of time.

Again, if we consider collision between $O$ and each point on $\mathcal{F}$; and consider the collection of all such values of $\alpha$, we find that the collision cone is now split into two cones, and is defined by $\alpha \epsilon\left[18.21^{\circ}, 97.65^{\circ}\right] \cup\left[207.27^{\circ}, 216.88^{\circ}\right]$. See Fig. 3(c). We call this a split collision cone.

Lemma 3: If two point objects $A$ and $B$ are moving with constant velocities $\overrightarrow{V_{A}}$ and $\overrightarrow{V_{B}}$ (Fig. 1), and the initial position of only $A$ is fixed; then, as long as $\overrightarrow{V_{A}} \neq \overrightarrow{V_{B}}$, there exists a $\theta$ for which $A$ is on a collision course with $B$.

Proof: Let $z=V_{\theta}+i V_{r}$. Then, the collision condition (indicated in Lemma 2 ) is defined by $\arg z=3 \pi / 2$.

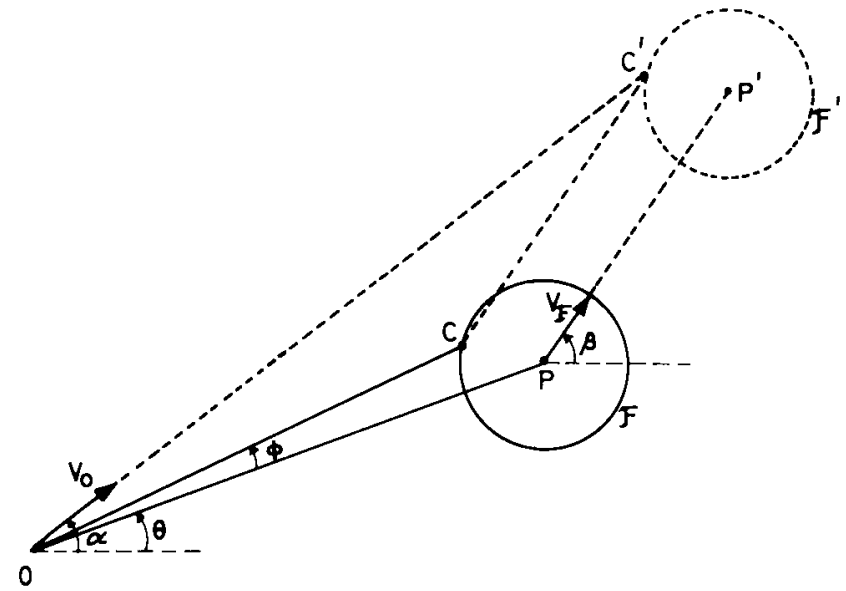

(a)

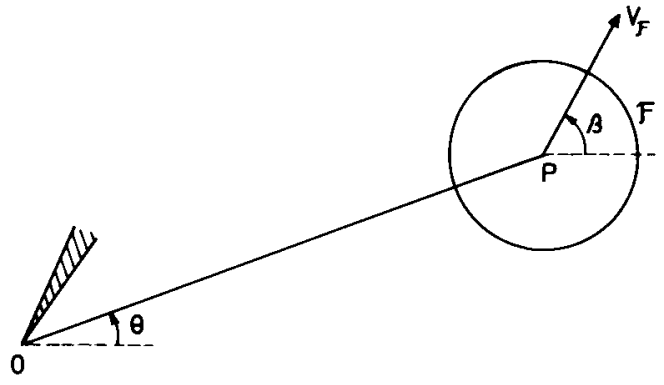

(b)

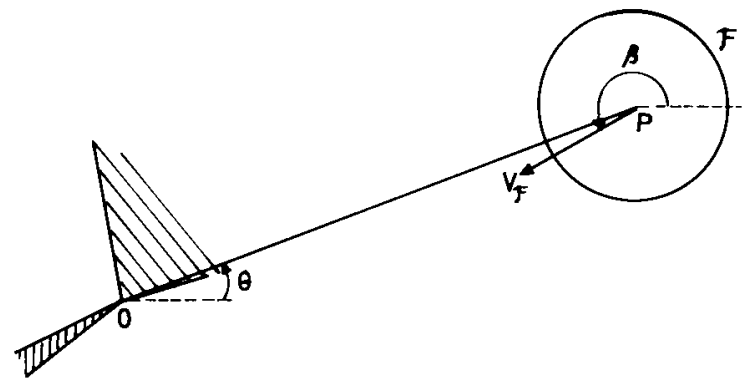

(c)

Fig. 3. (a) Collision geometry for Example 1, (b) collision cone for Example 1 , and (c) collision cone for Example 2.

Substituting for $V_{\theta}$ and $V_{r}$ from (1) and (2), we get

$$
z=e^{-i(\theta+(\pi / 2))}\left(V_{B} e^{i \beta}-V_{A} e^{i \alpha}\right)
$$

Therefore,

$$
\begin{aligned}
\arg z=\frac{3 \pi}{2} & \Rightarrow e^{-i(\theta+(\pi / 2))} \arg \left(V_{B} e^{i \beta}-V_{A} e^{i \alpha}\right)=\frac{3 \pi}{2} \\
& \Rightarrow \theta=\tan ^{-1} \frac{V_{B} \sin \beta-V_{A} \sin \alpha}{V_{B} \cos \beta-V_{A} \cos \alpha}
\end{aligned}
$$

Thus, from (9), as long as $\overrightarrow{V_{A}} \neq \overrightarrow{V_{B}}$, there exists a $\theta$ that satisfies the conditions for collision. When $\overrightarrow{V_{A}}=\overrightarrow{V_{B}}$, we get $V_{\theta}=0 ; V_{r}=0$, which is not a collision condition. 


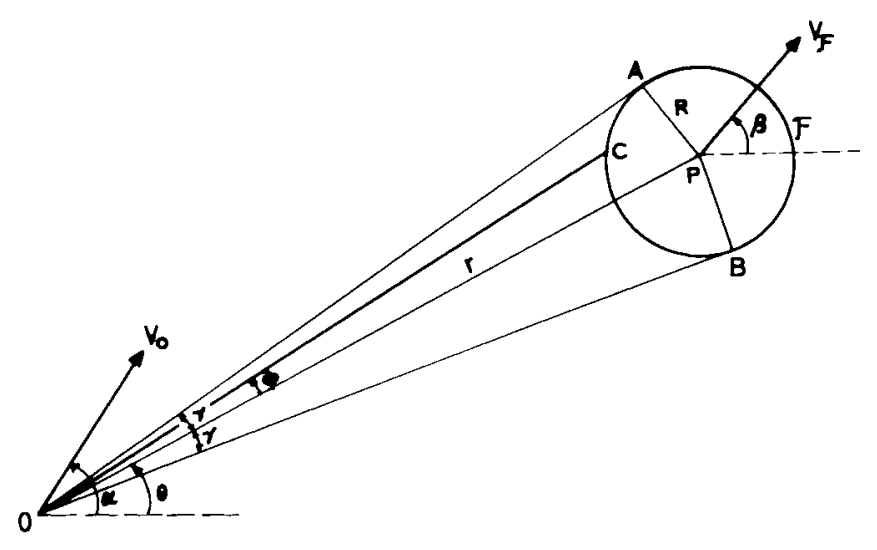

Fig. 4. Collision geometry between a point and a circle.

\section{The Collision CONE}

In the previous section, we illustrated the concept of a collision cone with the help of two examples. The collision cone can be used to predict the possibility of collision between two objects and to design collision avoidance strategies. In this section, we shall derive the necessary and sufficient conditions that enable the determination of the collision cone.

\section{A. Collision Between a Point and a Circular Object}

In Fig. 4, $O$ is a point object, while $\mathcal{F}$ is a circle of radius $R$ and with center at $P$. The velocities of $O$ and $\mathcal{F}$ are denoted by $V_{O}$ and $V_{\mathcal{F}}$, respectively. For any point $C$ (parametrized by the angle $\phi$ ) on the circle $\mathcal{F}$, we can write the relative velocity components of $O C$ as

$$
\begin{aligned}
& \left(V_{\theta}\right)_{O C}=V_{\mathcal{F}} \sin [\beta-(\theta+\phi)]-V_{O} \sin [\alpha-(\theta+\phi)] \\
& \left(V_{r}\right)_{O C}=V_{\mathcal{F}} \cos [\beta-(\theta+\phi)]-V_{O} \cos [\alpha-(\theta+\phi)] .
\end{aligned}
$$

Lemma 4: The point object $O$ is headed for a collision with the circle $\mathcal{F}$ if and only if there exists a ray $O C$, passing through $\mathcal{F}$, for which $\left(V_{\theta}\right)_{O C}=0 ;\left(V_{r}\right)_{O C}<0(C$ is called the collision point).

Proof: Follows from Lemma 2.

Now, let us define the relative velocity components of $O A$ (the upper tangent to the circle $\mathcal{F}$ from point $O$ ) as $\left(V_{\theta}\right)_{O A}$ and $\left(V_{r}\right)_{O A}$ and those of $O B$ (the lower tangent to the circle from point $O)$ as $\left(V_{\theta}\right)_{O B}$ and $\left(V_{r}\right)_{O B}$. These expressions are obtained from (10) and (11) by substituting $\phi=\gamma$ and $\phi=-\gamma$, respectively.

Lemma 5: At any given time, if $\left(V_{\theta}\right)_{O A}\left(V_{\theta}\right)_{O B} \leq 0$, then there exists exactly one ray $O C$, where $C$ is a point on the circle $\mathcal{F}$, that satisfies $\left(V_{\theta}\right)_{O C}=0$.

Proof: Consider an arbitrary point $C$ on the circle $\mathcal{F}$, defined by the angle $\phi \epsilon[-\gamma, \gamma]$. From (10), it is obvious that $\left(V_{\theta}\right)_{O C}$ is a continuous function of $\phi$. Thus, $\left(V_{\theta}\right)_{O A}\left(V_{\theta}\right)_{O B} \leq$ 0 implies that there exists at least one $\phi$, for which $\left(V_{\theta}\right)_{O C}=$ 0 . It remains to be shown that this point is unique. Putting $\left(V_{\theta}\right)_{O C}=0$ in $(10)$, we get

$$
\tan \phi=\frac{V_{\mathcal{F}} \sin (\beta-\theta)-V_{O} \sin (\alpha-\theta)}{V_{\mathcal{F}} \cos (\beta-\theta)-V_{O} \cos (\alpha-\theta)} .
$$

Since $\tan \phi$ is a periodic function with period $\pi$, successive $\phi$ that satisfy $\left(V_{\theta}\right)_{O C}=0$ occur at intervals of $\pi$. The angle $\phi$ is always bounded in the sense that $\phi \epsilon[-\gamma, \gamma]$, where $\gamma=\sin ^{-1}\left(\begin{array}{l}R \\ r\end{array}\right)$. For $r \geq R$, we have $\gamma \in\left[0^{\circ}, 90^{\circ}\right]$, and obviously this interval is insufficient to allow for more than one value of $\phi$, for which $\left(V_{\theta}\right)_{O C}=0$.

We now convert the condition $\left(V_{\theta}\right)_{O A}\left(V_{\theta}\right)_{O B} \leq 0$ into an equivalent condition with reference to $O P$ for which $\left(V_{\theta}\right)_{O P}$ and $\left(V_{r}\right)_{O P}$ are obtained from (10) and (11) by putting $\phi=0$. Now, consider the inequality

$$
\left(V_{\theta}\right)_{O A}\left(V_{\theta}\right)_{O B} \leq 0 \text {. }
$$

Substituting the expressions for $\left(V_{\theta}\right)_{O A}$ and $\left(V_{\theta}\right)_{O B}$ by putting $\phi=\gamma$ and $\phi=-\gamma$, respectively in (10), we get

$$
\begin{aligned}
& V_{\mathcal{F}}^{2} \sin [\beta-(\theta+\gamma)] \sin [\beta-(\theta-\gamma)] \\
& -V_{O} V_{\mathcal{F}} \sin [\alpha-(\theta+\gamma)] \sin [\beta-(\theta-\gamma)] \\
& -V_{O} V_{\mathcal{F}} \sin [\alpha-(\theta-\gamma)] \sin [\beta-(\theta+\gamma)] \\
& +V_{O}^{2} \sin [\alpha-(\theta+\gamma)] \sin [\alpha-(\theta-\gamma)] \leq 0 \text {. }
\end{aligned}
$$

Substituting $\cos 2 \gamma=\left(r^{2}-2 R^{2}\right) / r^{2}$ and simplifying, we get,

$$
r^{2}\left(V_{\theta}\right)_{O P}^{2} \leq R^{2}\left\{\left(V_{r}\right)_{O P}^{2}+\left(V_{\theta}\right)_{O P}^{2}\right\} \text {. }
$$

We omit the subscript $O P$ in the above equation for convenience, and henceforth denote $\left(V_{\theta}\right)_{O P}$ and $\left(V_{r}\right)_{O P}$ simply by $V_{\theta}$ and $V_{r}$. Thus, (15) can be written as

$$
r^{2} V_{\theta}^{2} \leq R^{2}\left\{V_{r}^{2}+V_{\theta}^{2}\right\} .
$$

Lemma 6: If a point and a circle of radius $R$ are moving with constant velocities such that they satisfy (16) at any given instant in time, then they will continue to satisfy (16) for all future time.

Proof: Define a function $f(t)$ as

$$
f(t)=r^{2} V_{\theta}^{2}-R^{2}\left(V_{r}^{2}+V_{\theta}^{2}\right) .
$$

Differentiating (17) with respect to $t$, we get

$$
\frac{d f}{d t}=2\left(r^{2} \dot{V}_{\theta} V_{\theta}+r \dot{r} V_{\theta}^{2}\right)-2 R^{2}\left(\dot{V}_{r} V_{r}+\dot{V}_{\theta} V_{\theta}\right)
$$

Using (5), we get

$$
\frac{d f}{d t}=2\left(r^{2} \dot{V}_{\theta} V_{\theta}+r \dot{r} V_{\theta}^{2}\right)=2 r\left(r \dot{V}_{\theta} V_{\theta}+\dot{r} V_{\theta}^{2}\right) \text {. }
$$

Putting $\dot{V}_{\theta}=-\dot{\theta} V_{r}$ and $\dot{r}=V_{r}$ in (19), we get

$$
\frac{d f}{d t}=2 r\left(-r \dot{\theta} V_{r} V_{\theta}+\dot{r} V_{\theta}^{2}\right)=2 r\left(-V_{r} V_{\theta}^{2}+V_{r} V_{\theta}^{2}\right)=0 .
$$

Thus, since $f(t)$ remains unchanged with time, if $f(t)<0$ initially, it remains so for all future time.

Lemma 6 is thus a generalization of Lemma 1.

Consider Fig. 2 (where $V_{\theta}$ and $V_{r}$ are, in fact, $\left(V_{\theta}\right)_{O P}$ and $\left.\left(V_{r}\right)_{O P}\right)$. Consider a $\left(V_{\theta}, V_{r}\right)$ trajectory starting at some initial point $\left(V_{\theta 0}, V_{r 0}\right)$. Then, two cases are of interest.

Case 1: $V_{\theta 0}=0 ; V_{r 0}<0$.

This implies that the collision point $C$ lies on the line $O P$ (Fig. 4), i.e., $O C P$ is a straight line. So, $\left(V_{\theta 0}, V_{r 0}\right)$ is a stationary point on the $\left(V_{\theta}, V_{r}\right)$ plane (Lemma 1$)$. 
Case 2: $V_{\theta 0} \neq 0 ; V_{r 0}<0$.

In this case, a trajectory originating from the third (fourth) quadrant moves into the second (first) quadrant, crossing the $V_{r}=0$ line. At the crossing point $V_{r}=\dot{r}=0$, implying that $r$ is an extremum at that instant (say at time $t=t_{m}$ ). Since $V_{r}$ is negative for $t<t_{m}$ and positive for all $t>t_{m}, r$ is also a minimum at $t=t_{m}$. Let $r\left(t_{m}\right)=r_{m}$. We have to determine the conditions for which $r_{m} \leq R$, since this implies collision. Multiplying both sides of (4) by $r$, we get

$$
\dot{V}_{\theta} / V_{\theta}=-V_{r} / r=-\dot{r} / r
$$

which, on integration, yields

$$
r V_{\theta}=r_{0} V_{\theta 0} \Rightarrow r^{2} V_{\theta}^{2}=r_{0}^{2} V_{\theta 0}^{2} \Rightarrow r_{m}^{2} V_{\theta m}^{2}=r_{0}^{2} V_{\theta 0}^{2}
$$

where $V_{\theta m}=V_{\theta}\left(t_{m}\right)$. If the initial conditions are such that they satisfy $r_{0}^{2} V_{\theta 0}^{2} \leq R^{2}\left(V_{r 0}^{2}+V_{\theta 0}^{2}\right)$ then, from Lemma 6 , we know that even at $t=t_{m}$

$$
r_{m}^{2} V_{\theta m}^{2} \leq R^{2}\left(V_{r m}^{2}+V_{\theta m}^{2}\right) .
$$

But $V_{r m}=V_{r}\left(t_{m}\right)=0$. Therefore, $r_{m}^{2} \leq R^{2} \Rightarrow r_{m} \leq R$. These results automatically lead to the following theorem.

Theorem 1: If a point and a circle are moving with constant velocities such that their initial conditions satisfy

$$
r_{0}^{2} V_{\theta 0}^{2} \leq R^{2}\left(V_{r 0}^{2}+V_{\theta 0}^{2}\right) \text { and } V_{r 0}<0
$$

then they are headed for a collision. The above conditions are both necessary and sufficient for a collision to occur.

Proof: Follows from the analysis given above.

Putting $R=0$, (22) reduces to $V_{\theta 0}=0$ and $V_{r 0}<0$, which are the conditions for collision between two point objects (Lemma 2). Substituting $V_{\theta 0}$ and $V_{r 0}$ from (1) and (2) in (16), we get

$$
\begin{aligned}
& r_{0}^{2}\left\{V_{\mathcal{F}} \sin \left(\beta-\theta_{0}\right)-V_{O} \sin \left(\alpha-\theta_{0}\right)\right\}^{2} \\
& \quad \leq R^{2}\left\{V_{O}^{2}+V_{\mathcal{F}}^{2}-2 V_{O} V_{\mathcal{F}} \cos (\alpha-\beta)\right\}
\end{aligned}
$$

Equation (23) can be interpreted as follows. Given $r_{0}, \theta_{0}, V_{O}, V_{\mathcal{F}}, \beta$, and $R$, there exists a range of $\alpha$ that satisfy (23) and for which $V_{r 0}<0$. Let us denote this range by $\left[\alpha_{1}, \alpha_{2}\right]$. Now, if $O$ has a velocity vector at an angle $\alpha$ such that $\alpha \epsilon\left[\alpha_{1}, \alpha_{2}\right]$, then collision is inevitable. We call this cone, with vertex at $O$ and angular limits defined by $\alpha_{1}$ and $\alpha_{2}$, as the collision cone. To determine the collision cone we first rewrite (16) as

$$
V_{\theta 0}^{2} \leq p^{2} V_{r 0}^{2}
$$

where

$$
p=R / \sqrt{r_{0}^{2}-R^{2}} .
$$

Also, we define the following quantities:

$$
\eta=\alpha-\theta_{0} ; \mu=\beta-\theta_{0} ; \nu=\frac{V_{O}}{V_{\mathcal{F}}} .
$$

The collision cone comprises of those values of $\eta$ that satisfy (24) and yield $V_{r 0}<0$. We define a set $\mathcal{N}_{1}$ as

$$
\mathcal{N}_{1}=\left\{\eta: V_{r 0}<0\right\}
$$

and we rewrite (24) as

$$
p V_{r 0} \leq V_{\theta_{0}} \leq-p V_{r 0}
$$

We now define two more sets $\mathcal{N}_{21}$ and $\mathcal{N}_{22}$ as

$$
\begin{aligned}
& \mathcal{N}_{21}=\left\{\eta: V_{\theta 0} \leq-p V_{r 0}\right\} \\
& \mathcal{N}_{22}=\left\{\eta: p V_{r 0} \leq V_{\theta 0}\right\}
\end{aligned}
$$

The collision cone is then obtained as a set $\mathcal{N}$ defined as

$$
\mathcal{N}=\mathcal{N}_{1} \cap\left(\mathcal{N}_{21} \cap \mathcal{N}_{22}\right) .
$$

The set $\mathcal{N}_{1}$ is obtained through the following cases.

Case 1: If $(\cos \mu / \nu) \geq 1$, then $\mathcal{N}_{1}$ is null.

Case 2: If $-1 \leq(\cos \mu / \nu)<1$, then

$$
\mathcal{N}_{1}=\left\{\eta:-\cos ^{-1}\left(\frac{\cos \mu}{\nu}\right)<\eta<\cos ^{-1}\left(\frac{\cos \mu}{\nu}\right)\right\} .
$$

Case 3: If $(\cos \mu / \nu)<-1$, then $\mathcal{N}_{1}$ spans the whole space, i.e.,

$$
\mathcal{N}_{1}=\{\eta: 0 \leq \eta \leq 2 \pi\} .
$$

To determine $\mathcal{N}_{21}$ we rewrite $V_{\theta 0}<-p V_{r 0}$ as

$$
(p \cos \mu+\sin \mu) \leq \nu(p \cos \eta+\sin \eta) .
$$

Now, defining

$$
\begin{aligned}
\sin \zeta & =\frac{p}{\sqrt{p^{2}+1}} ; \quad \cos \zeta=\frac{1}{\sqrt{p^{2}+1}} ; \\
A & =\frac{p \cos \mu+\sin \mu}{\sqrt{p^{2}+1}}
\end{aligned}
$$

so that $\zeta$ is in the first quadrant, we may rewrite (34) as

$$
\sin (\zeta+\eta) \geq A / \nu
$$

We define the two values of $\eta$, that satisfy (36) with a strict equality, as $\eta_{1}$ and $\eta_{2}$. Then, $\mathcal{N}_{21}$ can be obtained from:

Case 1: If $A / \nu>1$, then $\mathcal{N}_{21}$ is null.

Case 2: If $0 \leq(A / \nu) \leq 1$, then $\eta_{2} \geq \eta_{1}$ and

$$
\mathcal{N}_{21}=\left\{\eta: \eta_{1} \leq \eta \leq \eta_{2}\right\}
$$

where

$$
\eta_{1}=\sin ^{-1}(A / \nu)-\zeta ; \quad \eta_{2}=\pi-\sin ^{-1}(A / \nu)-\zeta .
$$

Case 3: If $-1<(A / \nu)<0$, then $\eta_{1} \geq \eta_{2}$, and

$$
\mathcal{N}_{21}=\left\{\eta: \eta \geq \eta_{1}, \eta \leq \eta_{2}\right\}
$$

where

$$
\eta_{1}=\sin ^{-1}(A / \nu)-\zeta ; \eta_{2}=-\pi-\sin ^{-1}(A / \nu)-\zeta .
$$



i.e.,

Case 4: If $(A / \nu) \leq-1$, then $\mathcal{N}_{21}$ spans the whole space, i.e.,

$$
\mathcal{N}_{21}=\{\eta: 0 \leq \eta \leq 2 \pi\} .
$$

To determine $\mathcal{N}_{22}$, we rewrite $p V_{r 0} \leq V_{\theta 0}$ as

$$
(p \cos \mu-\sin \mu) \leq \nu(p \cos \eta-\sin \eta) .
$$

Define

$$
\begin{aligned}
\sin \tilde{\zeta} & =\frac{p}{\sqrt{p^{2}+1}} ; \quad \cos \tilde{\zeta}=\frac{-1}{\sqrt{p^{2}+1}} ; \\
\tilde{A} & =\frac{p \cos \mu-\sin \mu}{\sqrt{p^{2}+1}}
\end{aligned}
$$

so that $\tilde{\zeta}=\pi-\zeta$ lies in the second quadrant. From (42)

$$
\sin (\tilde{\zeta}+\eta) \geq \tilde{A} / \nu
$$

We define the values of $\eta$, that satisfy (44) with a strict equality, as $\tilde{\eta}_{1}$ and $\tilde{\eta}_{2}$. Then the set $\mathcal{N}_{22}$ is obtained from:

Case 1: If $(\tilde{A} / \nu)>1$, then $\mathcal{N}_{22}$ is null.

Case 2: If $0 \leq(\tilde{A} / \nu) \leq 1$, then $\tilde{\eta}_{2} \geq \tilde{\eta}_{1}$, and

$$
\mathcal{N}_{22}=\left\{\eta: \tilde{\eta}_{1} \leq \eta \leq \tilde{\eta}_{2}\right\}
$$

where

$$
\tilde{\eta}_{1}=\sin ^{-1}(\tilde{A} / \nu)-\tilde{\zeta} ; \tilde{\eta}_{2}=\pi-\sin ^{-1}(\tilde{A} / \nu)-\tilde{\zeta} .
$$

Case 3: If $-1<\left(\tilde{A} / \nu<0\right.$, then $\tilde{\eta}_{1} \geq \tilde{\eta}_{2}$, and

$$
\mathcal{N}_{22}=\left\{\eta: \eta \geq \tilde{\eta}_{1}, \eta \leq \tilde{\eta}_{2}\right\}
$$

where

$$
\tilde{\eta}_{1}=\sin ^{-1}(\tilde{A} / \nu)-\tilde{\zeta} ; \tilde{\eta}_{2}=-\pi-\sin ^{-1}(\tilde{A} / \nu)-\tilde{\zeta} .
$$

Case 4: If $(\tilde{A} / \nu) \leq-1$, then $\mathcal{N}_{22}=\{\eta: 0 \leq \eta \leq 2 \pi\}$.

The boundaries of $\mathcal{N}_{21} \cap \mathcal{N}_{22}$ are then determined as follows.

1) If $(A / \nu)>1$ and/or $(\tilde{A} / \nu)>1$ then $\mathcal{N}_{21} \cap \mathcal{N}_{22}$ is null.

2) If both $-1<(A / \nu) \leq 1$ and $-1<(\tilde{A} / \nu) \leq 1$, then

a) If $\nu>1$, and $\zeta \geq \frac{1}{2}\left|\sin ^{-1}(A / \nu)+\sin ^{-1}(\tilde{A} / \nu)\right|$, then

$$
\mathcal{N}_{21} \cap \mathcal{N}_{22}=\left\{\eta: \eta_{1} \leq \eta \leq \tilde{\eta}_{2}\right\} .
$$

b) If $\nu<1$, and $0 \leq \zeta \leq \frac{1}{2}\left\{\sin ^{-1}(A / \nu)+\right.$ $\left.\sin ^{-1}(\tilde{A} / \nu)\right\}$, then

$\mathcal{N}_{21} \cap \mathcal{N}_{22}=\left\{\eta: \eta_{1} \leq \eta \leq \tilde{\eta}_{2}\right\} \cup\left\{\eta: \tilde{\eta}_{1} \leq \eta \leq \eta_{2}\right\}$.

c) In all other cases, $\mathcal{N}_{21} \cap \mathcal{N}_{22}$ is null.

3) For all other cases,

a) If $(A / \nu) \leq-1$, and $-1<(\tilde{A} / \nu) \leq 1$, then $\mathcal{N}_{21} \cap$ $\mathcal{N}_{22}=\mathcal{N}_{22}$.

b) If $-1<(A / \nu) \leq 1$ and $(\tilde{A} / \nu) \leq-1$, then $\mathcal{N}_{21} \cap$ $\mathcal{N}_{22}=\mathcal{N}_{21}$.

c) If $(A / \nu) \leq-1$ and $(\tilde{A} / \nu) \leq-1$, then $\mathcal{N}_{21} \cap$ $\mathcal{N}_{22}=\{\eta: 0 \leq \eta \leq 2 \pi\}$.

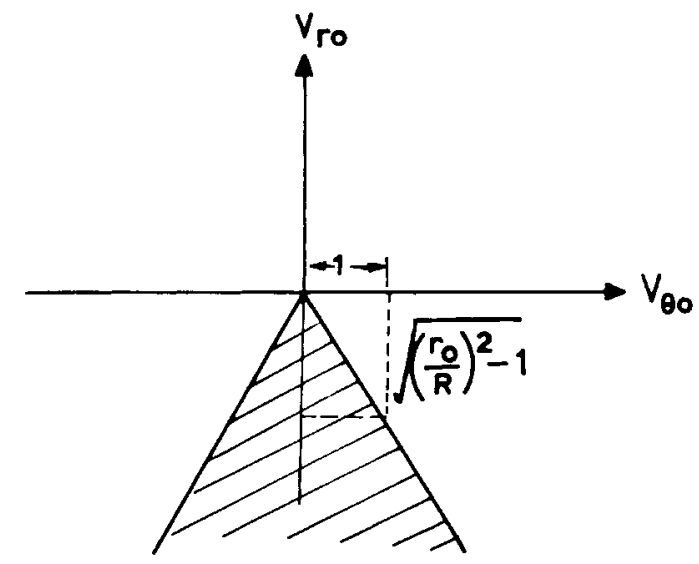

Fig. 5. Collision region in the $\left(V_{\theta 0}, V_{r 0}\right)$ plane.

Example 3: Let the initial geometry be identical to that in Example 1. This corresponds to $\nu=1.33, p=0.314, \mu=$ $15^{\circ}$. Since $(\cos \mu / \nu)=0.72$, we use (32) to obtain $\mathcal{N}_{1}=$ $\left\{\eta:-43.42^{\circ} \leq \eta \leq 43.42^{\circ}\right\}$. From (35), $\zeta=17.22^{\circ}$ and $A=0.53$. From (43), $\tilde{\zeta}=162.78^{\circ}$ and $\tilde{A}=0.038$. Since $\zeta>\frac{1}{2}\left|\sin ^{-1}(A / \nu)+\sin ^{-1}(\tilde{A} / \nu)\right|=12.6^{\circ}$ and $\nu>1,(49)$ should be used to determine $\mathcal{N}_{21} \cap \mathcal{N}_{22}$. Since $(A / \nu)=0.4$, from (38) we get $\eta_{1}=6.35^{\circ}$. Since $(\tilde{A} / \nu)=0.038$, from (46) we get $\tilde{\eta}_{2}=15.56^{\circ}$. Therefore, $\mathcal{N}_{21} \cap \mathcal{N}_{22}=\left\{\eta: 6.35^{\circ} \leq\right.$ $\left.\eta \leq 15.56^{\circ}\right\}$. Thus, from (31) $\mathcal{N}=\left\{\eta: 6.35^{\circ} \leq \eta \leq 15.56^{\circ}\right\}$, from which the collision cone is obtained as $51.35^{\circ} \leq \alpha \leq$ $60.56^{\circ}$.

Example 4: Let the initial geometry be identical to that in Example 2. This corresponds to $\nu=0.8, p=0.314, \mu=$ $170^{\circ}$. Since $(\cos \mu / \nu)=-1.23,(33)$ is used to determine $\mathcal{N}_{1}=\{\eta: 0 \leq \eta \leq 2 \pi\}$. From (35), $\zeta=17.22^{\circ}$ and $A=-0.129$. From (43), $\tilde{\zeta}=162.78^{\circ}$ and $\tilde{A}=-0.461$. Since $0<\zeta<\frac{1}{2}\left|\sin ^{-1}(A / \nu)+\sin ^{-1}(\tilde{A} / \nu)\right|=21.42^{\circ}$ and $\nu<1$, (50) should be used to determine $\mathcal{N}_{21} \cap \mathcal{N}_{22}$. Since $(A / \nu)=$ -0.156 , from (40) we get $\eta_{1}=-26.79^{\circ}$ and $\eta_{2}=-188.21^{\circ}$. Since $(\tilde{A} / \nu)=-0.5625$, from $(48)$ we get $\tilde{\eta}_{1}=-197.73^{\circ}$ and $\tilde{\eta}_{2}=52.65^{\circ}$. Therefore, $\mathcal{N}_{21} \cap \mathcal{N}_{22}=\left\{\eta:-26.79^{\circ} \leq\right.$ $\left.\eta \leq 52.65^{\circ}\right\} \cup\left\{\eta:-197.73^{\circ} \leq \eta \leq-188.21^{\circ}\right\}$. Consequently, from (31) the collision cone is obtained as $\left\{18.21^{\circ} \leq \alpha \leq 97.65^{\circ}\right\} \cup\left\{207.27^{\circ} \leq \alpha \leq 216.79^{\circ}\right\}$.

A representation of the collision cone in the $\left(V_{\theta 0}, V_{r 0}\right)$ space can be obtained by rearranging the terms in (22) to yield the initial conditions that lead to collision as

$$
-V_{r 0} \geq\left(\sqrt{\left(\frac{r_{0}}{R}\right)^{2}-1}\right)\left|V_{\theta_{0}}\right| \text { and } \quad V_{r 0}<0 .
$$

Equation (51) represents a sector-shaped region in the $\left(V_{\theta 0}, V_{r 0}\right)$ plane, and is shown in Fig. 5.

Equation (31) gives the collision cone between a point $O$ and a circle $\mathcal{F}$. Let $\mathcal{F}^{\circ}$ be an open circle containing all the points in the interior of $\mathcal{F}$. Then the collision cone between $O$ and $\mathcal{F}^{\circ}$ is the open cone obtained by removing the boundaries of the collision cone given by (31). 

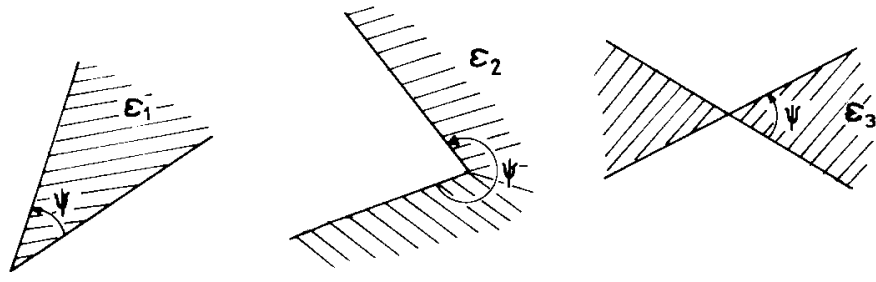

Fig. 6. Definition of cones $\mathcal{E}_{1}, \mathcal{E}_{2}, \mathcal{E}_{3}$.

\section{B. Collision Between a Point and an Irregularly Shaped Object}

It is possible to adapt Theorem 1 [and (31)] to obtain equivalent conditions that can be used to predict collision between a point and an irregularly shaped object. Before doing so, we give a few useful definitions [17]. A set $\mathcal{E}$ in a linear vector space is said to be a cone with vertex at the origin if $x \in \mathcal{E}$ implies that $\alpha x \in \mathcal{E}$ for all $\alpha \geq 0$. Fig. 6 shows three different types of cones $\mathcal{E}_{1}, \mathcal{E}_{2}$ and $\mathcal{E}_{3}$, each with vertex angle $\psi$. These cones differ from one another in the following manner: $\mathcal{E}_{1}$ is convex and has $\psi<\pi ; \mathcal{E}_{2}$ is nonconvex and has $\psi>\pi ; \mathcal{E}_{3}$ is nonconvex and has $\psi<\pi$. We shall use the notations $\mathcal{E}_{1}, \mathcal{E}_{2}$, and $\mathcal{E}_{3}$, respectively, to define these three different types of cones.

Consider Fig. 7(a) and (b), each of which shows the engagement geometry between a point object $A$ and an irregularly shaped object $\mathcal{B}$. We can construct a cone $\mathcal{E}$ with vertex at $A$, such that $\mathcal{E}$ is the smallest cone that contains the object $\mathcal{B}$, i.e., $\mathcal{E}$ is the intersection of all cones that contain $\mathcal{B}$ and have vertices at $A$. The vertex angle of $\mathcal{E}$ is denoted by $\psi$ and its boundaries are $A P$ and $A Q$. It can be seen that the cone in Fig. 7(a) is of the type $\mathcal{E}_{1}$ and that in Fig. 7(b) is of type $\mathcal{E}_{2}$. These cases are now considered separately.

Case 1: $\psi<\pi$.

We construct a circle $\mathcal{F}$ contained in $\mathcal{E}_{1}$ such that $A P$ and $A Q$ are tangential to $\mathcal{F}$. Let $\mathcal{F}$ have radius $R$ and center at a distance $r$ from $A$. Note that any choice of $R$ and $r$ should satisfy $R=r \sin (\psi / 2)$. This is illustrated in Fig. 7(a). Assuming that $\mathcal{F}$ moves with a velocity identical to that of $\mathcal{B}$, we can state the following lemma.

Lemma 7: If $\psi<\pi, A$ is on a collision course with $\mathcal{B}$ if and only if $A$ is on a collision course with $\mathcal{F}$.

Proof: From Lemma 4, for $A$ to be on a collision course with $\mathcal{F}$, it is both necessary and sufficient that there exists a ray $A C$, passing through $\mathcal{F}$, that has $\left(V_{\theta}\right)_{A C}=0 ;\left(V_{r}\right)_{A C}<0$. It can be seen that the ray $A C$ will always pass through $\mathcal{B}$, thus implying that $A$ is on a collision course with $\mathcal{B}$. This proves sufficiency.

The necessity is proved similarly. Let $A$ be on a collision course with $\mathcal{B}$. This implies that there exists a point $\mathrm{D}$ on $\mathcal{B}$, which is the point of collision [see Fig. 7(a)]. The ray $A D$ obviously must pass through $\mathcal{F}$.

As mentioned earlier, from Fig. 7(a), we can see that

$$
\sin (\psi / 2)=R / r
$$

For a given $\psi$, any circle $\mathcal{F}$ with radius $R$ and with center at a distance $r$ from A satisfying (52) will satisfy Lemma 7 .

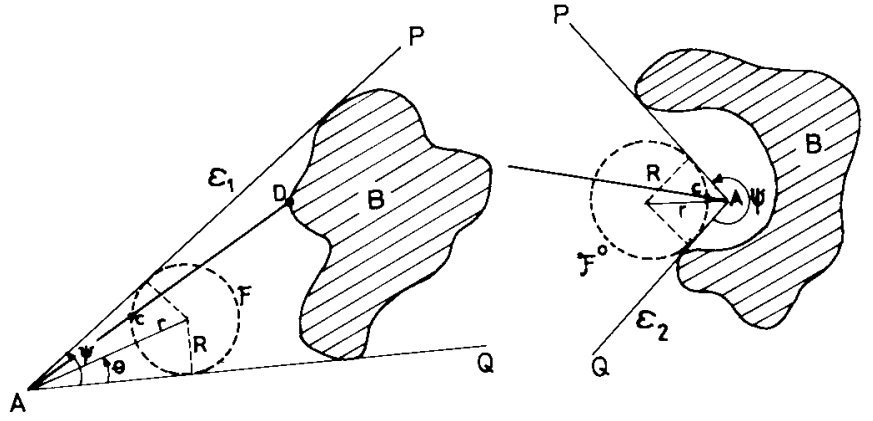

(a)

(b)

Fig. 7. Collision geometry between a point and an irregularly shaped object (a) $\psi<\pi$ and (b) $\psi>\pi$.

Case 2: $\psi>\pi$.

See Fig. 7(b). Here, we construct an open circle $\mathcal{F}^{\circ}$ contained in the complement of the cone $\mathcal{E}_{2}$ such that $A P$ and $A Q$ are tangential to the closure of $\mathcal{F}^{\circ}$. Note that $\mathcal{F}^{\circ}$ does not have any points in common with $A P$ or $A Q$.

Lemma 8: If $\psi>\pi$, then $\mathcal{A}$ is on a collision course with $\mathcal{B}$, if and only if $\mathcal{A}$ is not on a collision course with $\mathcal{F}^{\circ}$, and $\overrightarrow{V_{A}} \neq \overrightarrow{V_{B}}$.

Proof: To prove necessity, let $\mathcal{A}$ be on a collision course with $\mathcal{B}$. Then there exists a ray $A D$, passing through $\mathcal{B}$, that has $\left(V_{\theta}\right)_{A D}=0 ;\left(V_{r}\right)_{A D}<0$. Obviously, $A D$ can never pass through $\mathcal{F}^{\circ}$, thus implying that $\mathcal{A}$ is not on a collision course with $\mathcal{F}^{\circ}$.

Sufficiency is then proved as follows. From Lemma 3, as long as $\overrightarrow{V_{A}} \neq \overrightarrow{V_{B}}$, there exists a ray $A C$ that has $\left(V_{\theta}\right)_{A C}=$ $0 ;\left(V_{r}\right)_{A C}<0$. If $\mathcal{A}$ is not on a collision course with $\mathcal{F}^{\circ}$, then the ray $A C$ will not pass through $\mathcal{F}^{\circ}$. Since $\mathcal{F}^{\circ}$ lies in the complement of $\mathcal{E}_{2}$, therefore $A C$ will have to pass through some point lying in $\mathcal{E}_{2}$. It can be seen that $A C$ will then always pass through $\mathcal{B}$, thus implying that $\mathcal{A}$ is on a collision course with $\mathcal{B}$.

From Fig. 7(b), we see that

$$
\sin (\pi-\psi / 2)=R / r \Rightarrow \sin (\psi / 2)=R / r .
$$

For a given $\psi$, any circle $\mathcal{F}^{\circ}$ that satisfies (53) will satisfy Lemma 8. Substituting (52) [or (53)] in (22), we get

$$
\frac{V_{\theta_{0}}^{2}}{V_{\theta_{0}}^{2}+V_{r 0}^{2}} \leq \sin ^{2}\left(\frac{\psi_{0}}{2}\right) \quad \text { and } \quad V_{r 0}<0
$$

where $\psi_{0}$ represents the initial value of $\psi$. Equation (54) is necessary and sufficient condition for collision between $A$ and $\mathcal{F}$. Thus (54), with the inequality replaced by a strict inequality, is the necessary and sufficient condition for collision between $A$ and $\mathcal{F}^{\circ}$. These conditions can thus be used as necessary and sufficient condition for collision between $A$ and $\mathcal{B}$ (in the manner stated by Lemmas 7 and 8 ). Since these conditions are invariant with respect to the choice of the circles $\mathcal{F}$ or $\mathcal{F}^{\circ}$ (so long as (52) or (53) is satisfied), we can discard $\mathcal{F}$ or $\mathcal{F}^{\circ}$ entirely, and take $V_{\theta_{0}}$ and $V_{r 0}$ to represent the initial relative velocity components of the angular bisector of $\mathcal{E}_{1}$ for $\psi<\pi$; and of the angular bisector of the complement of $\mathcal{E}_{2}$, for $\psi>\pi$. 


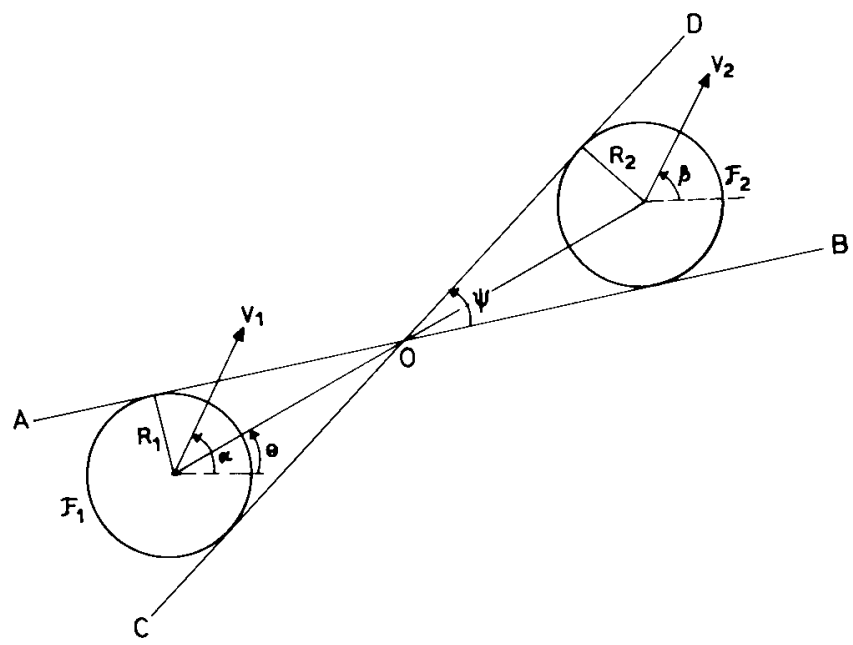

Fig. 8. Collision geometry between two circles.

The case when $\psi=\pi$ is a limiting case for both $\psi>\pi$ and $\psi<\pi$ and the corresponding collision cone can be obtained by using either Case 1 or Case 2 above with appropriate modifications for the limiting value.

Theorem 2: The collision cone between a point and an irregularly shaped object, both moving at constant velocities on a plane, is obtained as follows. Let $p$ in (35) and (43) be replaced by $\left|\tan \left(\psi_{0} / 2\right)\right|$. Now

1) If $\psi_{0} \leq \pi$ then the collision cone is obtained from (31), with $\theta_{0}$ as the initial angle of the angular bisector of the cone $\mathcal{E}_{1}$.

2) If $\psi_{0}>\pi$, then the collision cone is the complement of the cone obtained from (31) with $\theta_{0}$ as the initial angle formed by the angular bisector of the complement of the cone $\mathcal{E}_{2}$.

Proof: Follows from Lemmas 7 and 8, and the arguments given above.

Example 5: Let the values of $\theta_{0}, \beta, V_{O}$, and $V_{\mathcal{F}}$ be the same as in Example 2, but let the $\mathcal{F}$ now be replaced by some irregularly shaped object $\mathcal{B}$ such that $\psi=120^{\circ}$. Then from (31) and Theorem 2, we see that $O$ is headed for a collision with $\mathcal{B}$, if its velocity vector lies inside the angular bounds $\alpha \epsilon\left[31.42^{\circ}, 72.97^{\circ}\right]$. If $\psi=180^{\circ}$, then the collision cone is defined by $\alpha \epsilon\left[1.4^{\circ}, 88.57^{\circ}\right]$. If $\psi=240^{\circ}$, then the collision cone is defined by $\alpha \epsilon\left[72.97^{\circ}, 391.42^{\circ}\right]$.

\section{Collision Between Two Circular Objects}

Theorem 2 can be extended to enable detection of collision between two circular objects. Consider the engagement geometry in Fig. 8, where $\mathcal{F}_{1}$ and $\mathcal{F}_{2}$ are two circles of radii $R_{1}$ and $R_{2}$, moving with velocities $V_{1}$ and $V_{2}$, respectively. The lines $A B$ and $C D$ are the common tangents to the circles, intersecting at $O$. Note that these tangents form a cone of the type $\mathcal{E}_{3}$.

Theorem 3: The collision cone between two circles moving with constant velocities on a plane is given by (31) with $p$ in (35) and (43) equal to $\left|\tan \left(\psi_{0} / 2\right)\right|$, and $\theta_{0}$ as the initial angle of the line joining the centers of the two circles.

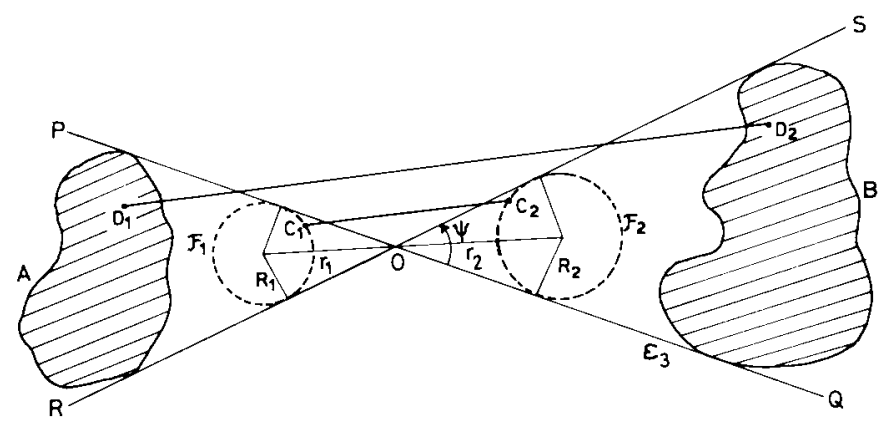

(a)

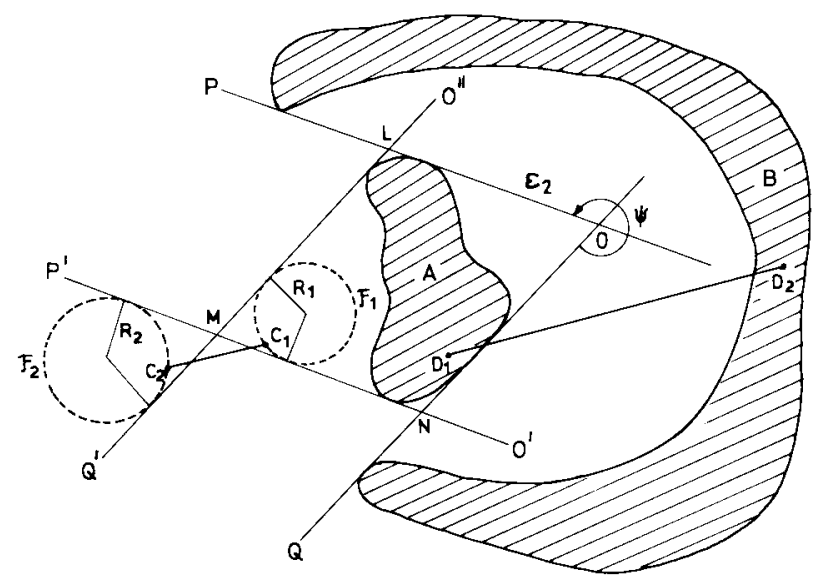

(b)

Fig. 9. Collision geometry between two irregularly shaped objects (a) $\psi<\pi$ and (b) $\psi>\pi$.

Proof: Superposing $\mathcal{F}_{1}$ onto $\mathcal{F}_{2}$, the problem reduces to one of collision prediction between a point and a circle of radius $\left(R_{1}+R_{2}\right)$. It is easy to see that the angle subtended by the enlarged circle $\mathcal{F}_{2}$ at the reduced point $\mathcal{F}_{1}$ [i.e., $\psi$ as defined in Fig. 7(a)] is equal to $\psi$ defined in Fig. 8. The proof then follows from 1) in Theorem 2.

\section{Collision Between Two Irregularly Shaped Objects}

We now extend Theorem 3 to predict collision between two irregularly shaped objects $\mathcal{A}$ and $\mathcal{B}$, moving with constant velocities on a plane. Note that when $\mathcal{A}$ and $\mathcal{B}$ are both irregularly shaped, it is difficult to superpose the shape of $\mathcal{A}$ onto that of $\mathcal{B}$. We therefore adopt a different approach. Consider the irregularly shaped objects as given in Fig. 9. Several possibilities then arise.

Case 1: $\psi<\pi$.

Refer Fig. 9(a). Here, the objects are well separated and we can construct a cone of the type $\mathcal{E}_{3}$ with the smallest vertex angle such that $\mathcal{A}$ and $\mathcal{B}$ are each contained on opposite sides of the vertex. Let $\psi$ denote the vertex angle, and $P Q$ and $R S$ be the boundaries that intersect at vertex $O$. We can then draw two circles, one contained in the cone $P O R$ and the other contained in the cone $S O Q$. Let $\mathcal{F}_{1}$ and $\mathcal{F}_{2}$ be these 
circles, of radii $R_{1}$ and $R_{2}$; with centers at a distance of $r_{1}$ and $r_{2}$, respectively, from $O$. This is illustrated in Fig. 9(a). If we now assume that $\mathcal{F}_{1}$ and $\mathcal{F}_{2}$ are moving with velocities identical to those of $\mathcal{A}$ and $\mathcal{B}$, respectively, then we have the following lemma.

Lemma 9: If $\psi<\pi, \mathcal{A}$ is on a collision course with $B$, if and only if $\mathcal{F}_{1}$ is on a collision course with $\mathcal{F}_{2}$.

Proof: If $\mathcal{F}_{1}$ is on a collision course with $\mathcal{F}_{2}$, then there exists a ray $C_{1} C_{2}$ (where $C_{1}$ is a point on $\mathcal{F}_{1}$ and $C_{2}$ is a point on $\mathcal{F}_{2}$ ), passing through both $\mathcal{F}_{1}$ and $\mathcal{F}_{2}$ and satisfying $\left(V_{\theta}\right)_{C_{1} C_{2}}=0 ;\left(V_{r}\right)_{C_{1} C_{2}}<0$. Now if $D_{1} D_{2}$ represents any ray parallel to $C_{1} C_{2}$, then it is evident that $\left(V_{\theta}\right)_{D_{1} D_{2}}=0 ;\left(V_{r}\right)_{D_{1} D_{2}}<0$. At least one such $D_{1} D_{2}$ ray (where $D_{1}$ is a point on $\mathcal{A}$ and $D_{2}$ is a point on $\mathcal{B}$ ) can be drawn, thus implying that $\mathcal{A}$ is on a collision course with $\mathcal{B}$. The necessity of the condition can be proved similarly.

Case 2: $\psi>\pi$.

In this case, we can construct a cone of type $\mathcal{E}_{2}$, such that

1) The boundaries of the cone touch both $\mathcal{A}$ and $\mathcal{B}$.

2) The whole of $\mathcal{B}$ is enclosed in the closed cone $\mathcal{E}_{2}$, and the whole of $\mathcal{A}$ is enclosed in the closure of the complement of $\mathcal{E}_{2}$. This is illustrated in Fig. 9(b).

Before we obtain the collision cone between $\mathcal{A}$ and $\mathcal{B}$, some construction is necessary [see Fig. 9(b)]. Draw lines $Q^{\prime} O^{\prime \prime}$ and $P^{\prime} O^{\prime}$ that are parallel to $Q O$ and $P O$, respectively, such that $\mathcal{A}$ is contained in the parallelogram $L M N O$. We can then draw two circles $\mathcal{F}_{1}$ and $\mathcal{F}_{2}$ such that $M N$ and $M L$ are tangential to $\mathcal{F}_{1}$; while $M P^{\prime}$ and $M Q^{\prime}$ are tangential to $\mathcal{F}_{2}$. Let $\mathcal{F}_{2}^{\circ}$ denote the open circle of $\mathcal{F}_{2}$. If we now assume that $\mathcal{F}_{1}$ and $\mathcal{F}_{2}^{\circ}$ are moving with velocities identical to those of $\mathcal{A}$ and $\mathcal{B}$, respectively, we can state the following lemma.

Lemma 10: If $\pi<\psi<2 \pi, \mathcal{A}$ is on a collision course with $\mathcal{B}$, if and only if $\mathcal{F}_{1}$ is not on a collision course with $\mathcal{F}_{2}^{\circ}$, and $\overrightarrow{V_{A}} \neq \overrightarrow{V_{B}}$.

Proof: Let $\mathcal{A}$ be on a collision course with $\mathcal{B}$. Then there exists a ray $D_{1} D_{2}$, (where $D_{1}$ is a point on $\mathcal{A}$ ), passing through $\mathcal{B}$, that has $\left(V_{\theta}\right)_{D_{1} D_{2}}=0 ;\left(V_{r}\right)_{D_{1} D_{2}}<0$. If $C_{1} C_{2}$ (where $C_{1}$ is a point on $\mathcal{F}_{1}$ ) denotes any general ray parallel to, and in the same direction as $D_{1} D_{2}$, then $\left(V_{\theta}\right)_{C_{1} C_{2}}=$ $0 ;\left(V_{r}\right)_{C_{1} C_{2}}<0$. Obviously, it is impossible to construct such a $C_{1} C_{2}$ ray that passes through $\mathcal{F}_{2}^{\circ}$, thus implying that $\mathcal{F}_{1}$ is not on a collision course with $\mathcal{F}_{2}^{\circ}$.

The sufficiency part is then proved as follows. We know from Lemma 3 that as long as $\overrightarrow{V_{A}} \neq \overrightarrow{V_{B}}$, there exists a ray $C_{1} C_{2}$ (where $C_{1}$ is a point on $\mathcal{F}_{1}$ ) such that $\left(V_{\theta}\right)_{C_{1} C_{2}}=$ $0 ;\left(V_{r}\right)_{C_{1} C_{2}}<0$. If $\mathcal{F}_{1}$ is not on a collision course with $\mathcal{F}_{2}^{\circ}$, then $C_{1} C_{2}$ can never pass through $\mathcal{F}_{2}^{\circ}$, and will therefore pass through any point lying in the complement of $\mathcal{F}_{2}^{\circ}$. Now, if $D_{1} D_{2}$ (where $D_{1}$ is a point on $\mathcal{A}$ ) denotes any general ray parallel to, and in the same direction as $C_{1} C_{2}$, then $\left(V_{\theta}\right)_{D_{1} D_{2}}=0 ;\left(V_{r}\right)_{D_{1} D_{2}}<0$. It can be seen that $D_{1} D_{2}$ will always pass through $\mathcal{B}$, thus implying that $\mathcal{A}$ is on a collision course with $\mathcal{B}$.

Theorem 4: The collision cone between two irregularly shaped objects $\mathcal{A}$ and $\mathcal{B}$, moving with constant velocities on a plane is obtained as follows: Let $p$ in (35) and (43) be equal to $\left|\tan \left(\psi_{0} / 2\right)\right|$. Now
1) If $0<\psi_{0} \leq \pi$, then the collision cone is obtained from (31), with $\theta_{0}$ as the initial angle formed by the angular bisector of the cone $\mathcal{E}_{3}$.

2) If $\pi<\psi_{0}<2 \pi$, then the collision cone is the complement of the cone obtained from (31), with $\theta_{0}$ as the initial angle formed by the angular bisector of the complement of the cone $\mathcal{E}_{2}$.

3) If $\psi_{0} \geq 2 \pi, \mathcal{A}$ will collide with $\mathcal{B}$ for any heading direction of $\mathcal{A}$ except when $\overrightarrow{V_{A}}=\overrightarrow{V_{B}}$.

Proof: From Lemmas 9, 10, and Theorem 3.

\section{Obstacle AVoidance}

The collision cone concept described thus far provides a convenient means to determine whether any two moving objects are on a collision course. The collision cone concept also helps to reduce the engagement between two irregularly shaped objects into an equivalent engagement between a point and a circle. In practice, the method by which the robot determines an imminent collision would depend on its onboard sensors. For example, suppose the robot can measure the relative velocity components with respect to the moving obstacle, then it can directly use (16) to predict a collision. However, note that it is not easy to obtain the relative velocities by using simple Doppler radars (as is done in aerospace applications) when the physical size of the objects are significant compared to the distance between them. In the case of automated vehicles in a factory environment, relative velocities may be obtained by using an overhead sensor to track the vehicle movements and transmit information to the robot [18]. Alternatively, if the robot is equipped with an inertial platform which provides it with its own velocity information, and radar sensors which provide it with the obstacles' instantaneous velocity information then the results given in Section III can be directly used to obtain the collision cone and thus predict an imminent collision.

If a robot is headed for a collision with some object in its environment (i.e., the robot's velocity vector lies inside the collision cone), it can adopt any of the following three strategies to avert collision.

1) The robot can maintain its heading direction constant; but change its speed so as to make its velocity vector lie outside the collision cone. This is equivalent to the robot speeding up, slowing down, or reversing to avoid colliding with the obstacle.

2) The robot can keep its speed constant; but change its heading direction until its velocity vector lies outside the collision cone. This is equivalent to the robot turning away from its original path.

3) The robot can change both its speed and its heading direction until its velocity vector lies outside the collision cone.

For 1) the robot has to apply a longitudinal acceleration (i.e., an acceleration along its heading direction); for 2) it has to apply a lateral acceleration (i.e., an acceleration perpendicular to its heading direction); while for 3) both longitudinal and lateral acceleration are required. The precise strategy to adopt would depend on the longitudinal/lateral acceleration limits of 
the robot, its kinematic constraints, and the time within which the robot should pull its heading out of the collision cone.

From the preceding discussion, it is evident that the range of speeds of the robot that will make its current heading direction lie outside the collision cone is given by $V_{O} \in \mathcal{S} \subseteq \Re$, where $\mathcal{S}$ is defined as

$$
\begin{aligned}
\mathcal{S} & =\mathcal{S}_{1} \cup \mathcal{S}_{2} \\
\mathcal{S}_{1} & =\left\{V_{O}: ; V_{\theta}^{2}>p^{2} V_{r}^{2}\right\} \\
\mathcal{S}_{2} & =\left\{V_{O}: V_{r}>0\right\} .
\end{aligned}
$$

We first determine the set $\mathcal{S}_{1}$. For $\tan ^{2} \eta-p^{2} \neq 0$, the equation $V_{\theta}^{2}-p^{2} V_{r}^{2}=0$ is quadratic in $V_{O}$. Denoting its roots by $V_{O 1}$ and $V_{O 2}$, we find that,

$$
\begin{aligned}
& V_{O 1}=V_{\mathcal{F}}\left(\frac{p \cos \mu+\sin \mu}{p \cos \eta+\sin \eta}\right) \\
& V_{O 2}=V_{\mathcal{F}}\left(\frac{p \cos \mu-\sin \mu}{p \cos \eta-\sin \eta}\right)
\end{aligned}
$$

where $\mu, \eta$, and $p$ are as defined in (25) and (26).

For $\tan ^{2} \eta-p^{2}=0$, the equation $V_{\theta}^{2}-p^{2} V_{r}^{2}=0$ is linear in $V_{O}$, and its root is denoted by $V_{O}^{*}$. Let

$$
\begin{aligned}
V_{O 1}^{*} & =V_{\mathcal{F}}\left(\frac{\sqrt{p^{2}+1}}{2 p}\right)(p \cos \mu+\sin \mu) \\
V_{O 2}^{*} & =V_{\mathcal{F}}\left(\frac{\sqrt{p^{2}+1}}{2 p}\right)(p \cos \mu-\sin \mu) \\
V_{O 3}^{*} & =-V_{O 1}^{*} ; V_{O 4}^{*}=-V_{O 2}^{*} \\
\mathcal{S}_{1}^{1} & =\left\{V_{O}: V_{O}>V_{O}^{*}\right\} \quad \mathcal{S}_{1}^{2}=\left\{V_{O}: V_{O}<V_{O}^{*}\right\} .
\end{aligned}
$$

Now, $\mathcal{S}_{1}$ can be obtained as follows.

Case 1: If $0<(\alpha-\beta)<\pi$, and

1) if $\tan ^{2} \eta-p^{2}>0$, then $V_{O 1}>V_{O 2}$, and

$$
\mathcal{S}_{1}=\left\{V_{O}: V_{O}<V_{O 2} \text { or } V_{O}>V_{O 1}\right\}
$$

2) if $\tan ^{2} \eta-p^{2}<0$, then $V_{O 1}<V_{O 2}$, and

$$
\mathcal{S}_{1}=\left\{V_{O}: V_{O 1}<V_{O}<V_{O 2}\right\} .
$$

Case 2: If $\pi<(\alpha-\beta)<2 \pi$, and

1) if $\tan ^{2} \eta-p^{2}>0$, then $V_{O 1}<V_{O 2}$, and

$$
\mathcal{S}_{1}=\left\{V_{O}: V_{O}>V_{O 2} \text { or } V_{O}<V_{O 1}\right\}
$$

2) if $\tan ^{2} \eta-p^{2}<0$, then $V_{O 1}>V_{O 2}$, and

$$
\mathcal{S}_{1}=\left\{V_{O}: V_{O 2}<V_{O}<V_{O 1}\right\} .
$$

Case 3: If $\sin (\alpha-\beta)=0$, and

1) if $\tan ^{2} \eta-p^{2}>0$, then $\mathcal{S}_{1}$ includes all values of $V_{O}$.

2) if $\tan ^{2} \eta-p^{2}<0$, then $\mathcal{S}_{1}$ is null.

Case 4: If $\eta=\tan ^{-1} p$, then $V_{O}^{*}=V_{O 1}^{*}$ and

1) if $p \cos \mu-\sin \mu>0$, then $\mathcal{S}_{1}=\mathcal{S}_{1}^{1}$.

2) if $p \cos \mu-\sin \mu<0$, then $\mathcal{S}_{1}=\mathcal{S}_{1}^{2}$.

3) if $p \cos \mu-\sin \mu=0$, then $\mathcal{S}_{1}$ is null.

Case 5: If $\eta=\pi+\tan ^{-1} p$, then $V_{O}^{*}=V_{O 3}^{*}$ and

1) if $p \cos \mu-\sin \mu>0$, then $\mathcal{S}_{1}=\mathcal{S}_{1}^{2}$.

2) if $p \cos \mu-\sin \mu<0$, then $\mathcal{S}_{1}=\mathcal{S}_{1}^{1}$.

3) if $p \cos \mu-\sin \mu=0$, then $\mathcal{S}_{1}$ is null.
Case 6: If $\eta=\pi-\tan ^{-1} p$, then $V_{O}^{*}=V_{O 4}^{*}$ and

1) if $p \cos \mu+\sin \mu>0$, then $\mathcal{S}_{1}=S_{1}^{2}$.

2) if $p \cos \mu+\sin \mu<0$, then $\mathcal{S}_{1}=S_{1}^{1}$.

3) if $p \cos \mu+\sin \mu=0$, then $\mathcal{S}_{1}$ is null.

Case 7: If $\eta=-\tan ^{-1} p$, then $V_{O}^{*}=V_{O 2}^{*}$ and

1) if $p \cos \mu+\sin \mu>0$, then $\mathcal{S}_{1}=\mathcal{S}_{1}^{1}$.

2) if $p \cos \mu+\sin \mu<0$, then $\mathcal{S}_{1}=\mathcal{S}_{1}^{2}$.

3) if $p \cos \mu+\sin \mu=0$, then $\mathcal{S}_{1}$ is null.

The set $\mathcal{S}_{2}$ can be determined from the following.

Case 1: If $\cos \eta>0$, then

$$
\mathcal{S}_{2}=\left\{V_{O}: V_{O}<V_{\mathcal{F}}(\cos \mu / \cos \eta)\right\} \text {. }
$$

Case 2: If $\cos \eta<0$, then

$$
\mathcal{S}_{2}=\left\{V_{O}: V_{O}>V_{\mathcal{F}}(\cos \mu / \cos \eta)\right\}
$$

Finally, from the above, the set $\mathcal{S}$ is obtained as follows.

Case 1: For $0<(\alpha-\beta)<\pi$

1) If $\tan ^{-1} p<\eta<\pi-\tan ^{-1} p$, then $\mathcal{S}=\Re$.

2) If $\pi+\tan ^{-1} p<\eta<-\tan ^{-1} p$, then $\mathcal{S}=\mathcal{S}_{1}$ in (60).

3) If $-\tan ^{-1} p<\eta<\tan ^{-1} p$, then $\mathcal{S}=\left\{V_{O}: V_{O}<V_{O 2}\right\}$.

4) If $\pi-\tan ^{-1} p<\eta<\pi+\tan ^{-1} p$, then $\mathcal{S}=$ $\left\{V_{O}: V_{O}>V_{O 1}\right\}$.

Case 2: For $\pi<(\alpha-\beta)<2 \pi$

1) If $\pi+\tan ^{-1} p<\eta<-\tan ^{-1} p$, then $\mathcal{S}=\Re$.

2) If $\tan ^{-1} p<\eta<\pi-\tan ^{-1} p$, then $\mathcal{S}=\mathcal{S}_{1}$ in (62).

3) If $-\tan ^{-1} p<\eta<\tan ^{-1} p$, then $\mathcal{S}=\left\{V_{O}: V_{O}<V_{O 1}\right\}$.

4) If $\pi-\tan ^{-1} p<\eta<\pi+\tan ^{-1} p$, then $\mathcal{S}=$ $\left\{V_{O}: V_{O}>V_{O 2}\right\}$.

Case 3: If $\sin (\alpha-\beta)=0$, and

1) if $\tan ^{2} \eta-p^{2}>0$, then $\mathcal{S}=\Re$.

2) if $\tan ^{2} \eta-p^{2}<0$, then $\mathcal{S}=\mathcal{S}_{2}$.

Case 4: If $\eta=\tan ^{-1} p$, and

1) if $p \cos \mu-\sin \mu>0$, then $\mathcal{S}=\Re$.

2) if $p \cos \mu-\sin \mu<0$, then $\mathcal{S}=\mathcal{S}_{1}$.

3) if $p \cos \mu-\sin \mu=0$, then $\mathcal{S}=\mathcal{S}_{2}$.

Case 5: If $\eta=\pi+\tan ^{-1} p$, and

1) if $p \cos \mu-\sin \mu<0$, then $\mathcal{S}=\mathcal{S}_{1}$.

2) if $p \cos \mu-\sin \mu \geq 0$, then $\mathcal{S}=\mathcal{S}_{2}$.

Case 6: If $\eta=\pi-\tan ^{-1} p$, and

1) if $p \cos \mu+\sin \mu<0$, then $\mathcal{S}=\mathcal{S}_{1}$.

2) if $p \cos \mu+\sin \mu \geq 0$, then $\mathcal{S}=\mathcal{S}_{2}$.

Case 7: If $\eta=-\tan ^{-1} p$, and

1) if $p \cos \mu+\sin \mu>0$, then $\mathcal{S}=\Re$.

2) if $p \cos \mu+\sin \mu<0$, then $\mathcal{S}=\mathcal{S}_{1}$.

3) if $p \cos \mu+\sin \mu=0$, then $\mathcal{S}=\mathcal{S}_{2}$.

The detailed derivations for the above results are available in [4].

Example 6: Consider the initial geometry as in Example 1. Let the robot have a heading direction defined by $\alpha=57^{\circ}$. Then, with its present speed $V_{O}=2$, it is headed for a collision with the obstacle. To determine the speed the robot must attain (while maintaining $\alpha=57^{\circ}$ ), we proceed as follows. For this geometry, $\eta=12^{\circ}, p=0.314, \mu=15^{\circ}, \tan ^{2} \eta-$ $p^{2}=-0.0534$. Therefore, from (58) and (59), we obtain 
$V_{O 1}=1.637$ and $V_{O 2}=0.672$. As $\alpha-\beta=-3^{\circ}$, we have $\pi<(\alpha-\beta)<2 \pi$ and $-\tan ^{-1} p<\eta<\tan ^{-1} p$. So, Case 2(3) above is used to determine $\mathcal{S}=\left\{V_{O}: V_{O}<1.637\right\}$. Therefore, if the robot reduces its speed to a value below 1.637, it can avoid a collision with the obstacle.

Example 7: Consider the initial geometry as in Example 2. Let the robot have a heading direction defined by $\alpha=80^{\circ}$. Then, with its present speed $V_{O}=2$, it is headed for a collision with the obstacle. For this geometry, $\eta=35^{\circ}, p=0.314, \mu=$ $-135^{\circ}, \tan ^{2} \eta-p^{2}=0.3916$. Therefore, from (58) and (59), we obtain $V_{O 1}=-0.408$ and $V_{O 2}=3.815$. As $\alpha-\beta=$ $225^{\circ}$, we have $\pi<(\alpha-\beta)<2 \pi$ and also $\tan ^{-1} p<\eta<\pi-$ $\tan ^{-1} p$. So, Case 2(2) above is used to determine $\mathcal{S}=$ $\left\{V_{O}: V_{O}<-0.408\right.$ or $\left.V_{O}>3.815\right\}$. Therefore, the robot must either increase its speed beyond 3.815 or reverse its direction and increase its speed beyond 0.408 , to avoid a collision with the obstacle.

\section{APPROXIMATING IRREGULARLy SHAPEd ObJECTS}

Use of the conditions of Theorem 4 to detect collision between irregularly shaped objects requires measurement of the angle $\psi$. This in turn requires construction of the cone $\mathcal{E}$, which could pose practical difficulties due to the arbitrary shapes of the objects under consideration. Therefore, when the measurement of $\psi$ is difficult, we propose another method. We can approximate the irregularly shaped objects $\mathcal{A}$ and $\mathcal{B}$ by a collection of circles each. Let $\mathcal{A}$ be approximated by $m$ circles $\mathcal{A}_{1}, \mathcal{A}_{2}, \cdots, \mathcal{A}_{m}$ and $\mathcal{B}$ be approximated by $n$ circles $\mathcal{B}_{1}, \mathcal{B}_{2}, \cdots, \mathcal{B}_{n}$. Then, by using Theorem 3 for collision between each of the $m$ circles of $\mathcal{A}$ with each of the $n$ circles of $\mathcal{B}$, we can obtain a total of $m n$ collision cones. The union of all these cones then can be meaningfully used to predict collision between the irregularly shaped objects $\mathcal{A}$ and $\mathcal{B}$. This type of approximation is standard in robot collision avoidance literature [5].

It is obvious that the success of this method depends on the choice of circles used to approximate $\mathcal{A}$ and $\mathcal{B}$. The collision cone thus obtained is inexact, in contrast to the exact collision cone that can be obtained using Theorem 4 . In the following analysis, we shall show that this inexact collision cone can still be used effectively for motion planning if the original objects are either both over-approximated or both under-approximated.

Examples of over- and under-approximation are shown in Fig. 10(a) and (b). Suppose $\mathcal{A}$ and $\mathcal{B}$ are the moving objects. Let $\mathcal{A}^{\prime}$ and $\mathcal{B}^{\prime}$ be the over-approximations and $\mathcal{A}^{\prime \prime}$ and $\mathcal{B}^{\prime \prime}$ be the under-approximations of these objects, respectively. If $\mathcal{N}(\mathcal{A}, \mathcal{B})$ represents the exact collision cone (obtained from Theorem 4), and $\mathcal{N}\left(\mathcal{A}^{\prime}, \mathcal{B}^{\prime}\right)$ and $\mathcal{N}\left(\mathcal{A}^{\prime \prime}, \mathcal{B}^{\prime \prime}\right)$ represent the collision cones obtained after over- and under-approximating, respectively, then

$$
\mathcal{N}\left(\mathcal{A}^{\prime \prime}, \mathcal{B}^{\prime \prime}\right) \subseteq \mathcal{N}(\mathcal{A}, \mathcal{B}) \subseteq \mathcal{N}\left(\mathcal{A}^{\prime}, \mathcal{B}^{\prime}\right)
$$

In the event of using circles to approximate irregularly shaped objects, it is difficult to obtain conditions that are both necessary and sufficient to predict collision. Over-approximation of $\mathcal{A}$ and $\mathcal{B}$ yields a collision cone that contains the exact collision cone. As a result, keeping the heading direction of

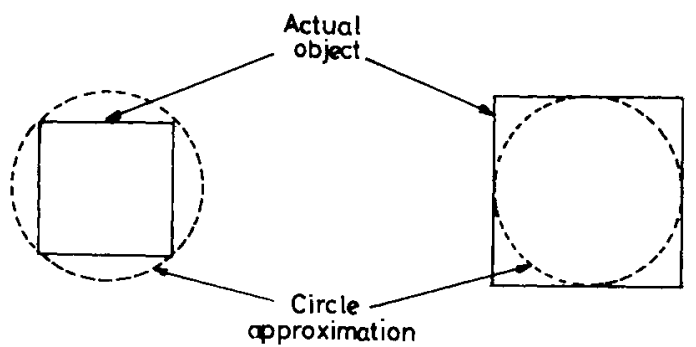

Fig. 10. Over- and under-approximations.

$\mathcal{A}$ outside such a cone is a sufficient condition for $\mathcal{A}$ to avoid $\mathcal{B}$. Similarly, since under-approximation of $\mathcal{A}$ and $\mathcal{B}$ yields a collision cone that is contained in the exact collision cone, keeping the heading direction of $\mathcal{A}$ outside such a cone becomes a necessary condition for $\mathcal{A}$ to avoid $\mathcal{B}$.

We now present three methods each of over- and underapproximating an object by circles. For this we define

$$
\mathcal{C}^{\mathcal{A}}=\bigcup_{i=1}^{m} \mathcal{A}_{i} \quad \mathcal{C}^{\mathcal{B}}=\bigcup_{i=1}^{n} \mathcal{B}_{i}
$$

\section{A. Over-Approximation}

Method 1: Choose the circles $\mathcal{A}_{i}$ and $\mathcal{B}_{i}$ such that

$$
\mathcal{A} \subseteq \mathcal{C}^{\mathcal{A}} \text { and } \quad \mathcal{B} \subseteq \mathcal{C}^{\mathcal{B}} .
$$

Method 2: Choose the circles $\mathcal{A}_{i}\left(\mathcal{B}_{i}\right)$ such that any straight line intersecting $\mathcal{A}(\mathcal{B})$ must also intersect $\mathcal{C}^{\mathcal{A}}\left(\mathcal{C}^{\mathcal{B}}\right)$. This condition implies that

$$
\mathcal{A} \subseteq \operatorname{Co}\left(\mathcal{C}^{\mathcal{A}}\right) \quad \text { and } \quad \mathcal{B} \subseteq \operatorname{Co}\left(\mathcal{C}^{\mathcal{B}}\right)
$$

where $\operatorname{Co}(X)$ denotes the convex hull of the set $X$. Note that (69) is automatically satisfied by the approximation adopted in Method 1, although an approximation using Method 2 may not satisfy (68). Thus Method 2 imposes a less stringent condition than Method 1.

Method 3: Choose the circles $\mathcal{A}_{i}$ and $\mathcal{B}_{i}$ such that any straight line intersecting both $\mathcal{A}$ and $\mathcal{B}$ must also intersect both $\mathcal{C}^{\mathcal{A}}$ and $\mathcal{C}^{\mathcal{B}}$. Note that this condition is automatically satisfied by the approximations adopted in Methods 1 and 2 . Thus Method 3 imposes a condition that is less stringent than either Methods 1 or 2.

Thus, if we denote the exact collision cone by $\mathcal{N}_{E}$, and the cones obtained by Methods 1,2 , and 3 as $\mathcal{N}_{1}, \mathcal{N}_{2}$, and $\mathcal{N}_{3}$, respectively, then for every over-approximation according to Method 1, there exists an over-approximation according to Method 2 and an over-approximation according to Method 3, such that

$$
\mathcal{N}_{E} \subseteq \mathcal{N}_{3} \subseteq \mathcal{N}_{2} \subseteq \mathcal{N}_{1} .
$$

Note that approximations according to Methods 1 and 2 can be used independent of the configuration of the objects in space. But an approximation according to Method 3 is valid only for a specific configuration of the objects. 
TABLE I

OVER-APPROXIMATION: METHOD 1

\begin{tabular}{l|c|c|c|c}
\hline Pairs & $r_{o}$ & $\theta_{0}$ & $\psi_{0}$ & Collision Cone \\
\hline $\mathcal{A}_{1}, \mathcal{B}_{1}$ & 12.91 & $-2.22^{\circ}$ & $20.39^{\circ}$ & $\alpha c\left[25.13^{\circ} .18 .18^{\circ}\right]$ \\
$\mathcal{A}_{1}, \mathcal{B}_{2}$ & 16.41 & $-1.75^{\circ}$ & $15.91^{\circ}$ & $\alpha \in\left[29.11^{\circ}, 16.71^{\circ}\right]$ \\
$\mathcal{A}_{2}, \mathcal{B}_{1}$ & 10.50 & $-1.09^{\circ}$ & $24.76^{\circ}$ & $\alpha c\left[22.55^{\circ} .50 .05^{\circ}\right]$ \\
$\mathcal{A}_{2}, \mathcal{B}_{2}$ & 14.00 & $-0.82^{\circ}$ & $18.31^{\circ}$ & $\alpha \in\left[28.30^{\circ} .17 .94^{\circ}\right]$ \\
$\mathcal{A}_{3}, \mathcal{B}_{1}$ & 12.32 & $10.76^{\circ}$ & $21.42^{\circ}$ & $\alpha \in\left[3.1 .38^{\circ}, 52.67^{\circ}\right]$ \\
$\mathcal{A}_{3}, \mathcal{B}_{2}$ & 15.77 & $8.38^{\circ}$ & $16.57^{\circ}$ & $\alpha\left[335.93^{\circ} .50 .17^{\circ}\right]$ \\
\hline
\end{tabular}

TABLE II

Over-APPROXIMATION: Method 2

\begin{tabular}{c|c|c|c|c}
\hline Pairs & $r_{o}$ & $\theta_{0}$ & $\psi_{0}$ & Collision Cone \\
\hline $\mathcal{A}_{1}, \mathcal{B}_{1}$ & 13.04 & $-4.44^{\circ}$ & $16.94^{\circ}$ & $\alpha \epsilon\left[26.51^{\circ}, 46.08^{\circ}\right]$ \\
$\mathcal{A}_{1}, \mathcal{B}_{2}$ & 17.03 & $-3.37^{\circ}$ & $12.89^{\circ}$ & $\alpha \epsilon\left[30.50^{\circ}, 44.87^{\circ}\right]$ \\
$\mathcal{A}_{2}, \mathcal{B}_{1}$ & 9.80 & $-1.75^{\circ}$ & $22.79^{\circ}$ & $\alpha \epsilon\left[23.73^{\circ}, 49.18^{\circ}\right]$ \\
$\mathcal{A}_{2}, \mathcal{B}_{2}$ & 13.80 & $-1.24^{\circ}$ & $15.97^{\circ}$ & $\alpha \epsilon\left[29.78^{\circ}, 46.92^{\circ}\right]$ \\
$\mathcal{A}_{3}, \mathcal{B}_{1}$ & 12.30 & $12.68^{\circ}$ & $17.97^{\circ}$ & $\alpha \epsilon\left[37.64^{\circ}, 52.22^{\circ}\right]$ \\
$\mathcal{A}_{3}, \mathcal{B}_{2}$ & 16.23 & $9.57^{\circ}$ & $13.54^{\circ}$ & $\alpha \epsilon\left[38.40^{\circ}, 49.87^{\circ}\right]$ \\
\hline
\end{tabular}

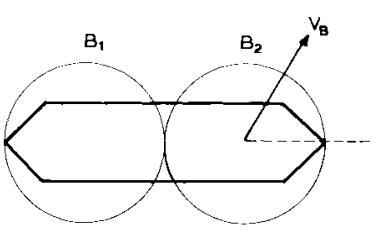

TABLE III

OVER-APPROXIMATION: METHOD 3

\begin{tabular}{c|c|c|c|c}
\hline Pairs & $r_{o}$ & $\theta_{0}$ & $\psi_{0}$ & Collision Cone \\
\hline $\mathcal{A}_{1}, \mathcal{B}_{1}$ & 11.02 & $-3.64^{\circ}$ & $20.16^{\circ}$ & $\alpha\left[\left[21.10^{\circ}, 17.59^{\circ}\right]\right.$ \\
$\mathcal{A}_{2}, \mathcal{B}_{1}$ & 11.07 & $6.22^{\circ}$ & $20.08^{\circ}$ & $\alpha\left[\left[32.22^{\circ}, 50.89^{\circ}\right]\right.$ \\
\hline
\end{tabular}

Example 8: Consider an engagement between two irregularly shaped objects $\mathcal{A}$ and $\mathcal{B}$ shown in Fig. 11(a), with $V_{A}=2, V_{B}=1.5, \beta=60^{\circ}, \theta_{0}=0^{\circ}$ and $\psi=40^{\circ}$. Then, by using the conditions of Theorem 4 , the exact collision cone $\mathcal{N}_{E}$ is given by $\alpha \epsilon\left[27.48^{\circ}, 49.72^{\circ}\right]$. If we now over-approximate $\mathcal{A}$ and $\mathcal{B}$ by each of the three methods discussed in the above section, we get the following results.

Method 1: Refer to Fig. 11(a). We approximate $\mathcal{A}$ by three circles $\mathcal{A}_{1}, \mathcal{A}_{2}$ and $\mathcal{A}_{3}$ of radii $2.3,2.2$, and 2.3 units and $\mathcal{B}$ by two circles $\mathcal{B}_{1}$ and $\mathcal{B}_{2}$ of radii 2.2 units each. The total number of $\left(\mathcal{A}_{i}, \mathcal{B}_{j}\right)$ pairs is thus six (see Table I). Taking a union of all the six collision cones, the final collision cone $\mathcal{N}_{1}$ is found to be $\alpha \epsilon\left[22.55^{\circ}, 52.67^{\circ}\right]$.

Method 2: Refer to Fig. 11(b). We approximate $\mathcal{A}$ by three circles $\mathcal{A}_{1}, \mathcal{A}_{2}, \mathcal{A}_{3}$ of radii $1.8 \mathrm{each}$; and $\mathcal{B}$ by two circles $\mathcal{B}_{1}, \mathcal{B}_{2}$ of radii 2 each. Again, six $\left(\mathcal{A}_{i}, \mathcal{B}_{j}\right)$ pairs are formed (see Table II). Taking a union of all the six collision cones, the final collision cone $\mathcal{N}_{2}$ is found to be $\alpha \epsilon\left[23.73^{\circ}, 52.22^{\circ}\right]$.

Method 3: Refer to Fig. 11(c). In this case, we approximate $\mathcal{A}$ by two circles $\mathcal{A}_{1}, \mathcal{A}_{2}$ of radii 2.1 each; and $\mathcal{B}$ by a single circle $\mathcal{B}_{1}$ of radius 1.7. The total number of $\left(\mathcal{A}_{i}, \mathcal{B}_{j}\right)$ pairs is thus two (see Table III). Taking a union of the two collision cones, the final collision cone $\mathcal{N}_{3}$ is found to be $\alpha \epsilon\left[24.40^{\circ}, 50.89^{\circ}\right]$.

It is seen that (70) is satisfied. By choosing a larger number of circles to approximate $\mathcal{A}$ and $\mathcal{B}$, an even closer approximation to the exact collision cone can be obtained.

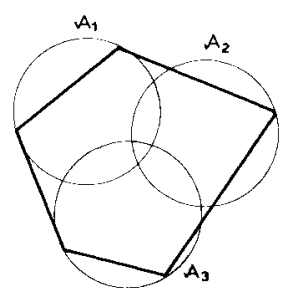

(b)

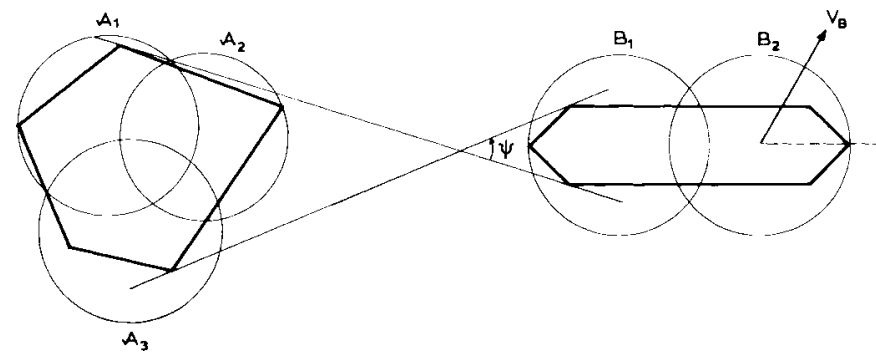

(a)

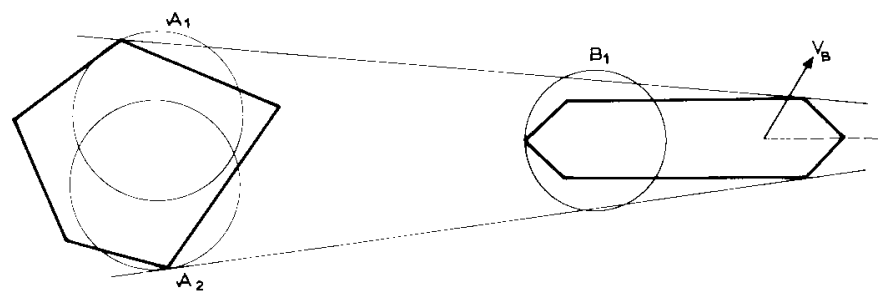

(c)

Fig. 11. Example 8 (a) Method 1, (b) Method 2, and (c) Method 3.

\section{B. Under-Approximation}

Method 1: Choose the circles $\mathcal{A}_{i}$ and $\mathcal{B}_{i}$ such that

$$
\mathcal{C}^{\mathcal{A}} \subseteq A \text { and } \quad \mathcal{C}^{\mathcal{B}} \subseteq B
$$

Method 2: Any straight line intersecting $\mathcal{C}^{\mathcal{A}}\left(\mathcal{C}^{\mathcal{B}}\right)$ must also intersect $\mathcal{A}(\mathcal{B})$. This implies that

$$
\mathcal{C}^{\mathcal{A}} \subseteq \operatorname{Co}(\mathcal{A}) \text { and } \quad \mathcal{C}^{\mathcal{B}} \subseteq \operatorname{Co}(\mathcal{B})
$$

Note that (72) is automatically satisfied by (71), but the converse is not true. Hence, Method 2 imposes a less stringent condition than Method 1.

Method 3: Choose the circles $\mathcal{A}_{i}$ and $\mathcal{B}_{i}$ such that any straight line intersecting both $\mathcal{C}^{\mathcal{A}}$ and $\mathcal{C}^{\mathcal{B}}$ must also intersect both $A$ and $B$. Note that this condition is automatically satisfied by the conditions used in Methods 1 and 2, although the converse is not true. Thus, method 3 imposes a less stringent condition than either of Methods 1 and 2.

As in the case of over-approximation, for every underapproximation according to Method 1, there exists an under-approximation according to Method 2 and an underapproximation according to Method 3, such that

$$
\mathcal{N}_{3} \subseteq \mathcal{N}_{2} \subseteq \mathcal{N}_{1} \subseteq \mathcal{N}_{E}
$$

Again, approximations according to Methods 1 and 2 can be used independent of the configuration of the objects in space, 
whereas an approximation according to Method 3 is valid only for a specific configuration of the objects.

\section{CONCLUSIONS}

Most of the earlier literature on collision avoidance strategies were restricted to static environments. Although there has been considerable interest in recent times on the more realistic dynamic environment, even these impose restrictions on the shapes of the robot and the obstacle (i.e., they are assumed to have some regular shapes such as circles or convex polygons). In this paper, we have relaxed the assumptions on static environments, and on the regularity of the obstacles' and the robot's shapes, and proposed the collision cone as an aid to collision detection and avoidance. An important contribution of this paper is that well-known results from guidance theory in the aerospace literature have been suitably modified and extended to yield concepts directly useful in the context of robot collision avoidance problems. These results are generalized to enable collision prediction between two irregularly shaped moving objects. Using the collision cone concept, some simple strategies by which collision can be avoided, are discussed.

This paper gives a preliminary—but fairly in-depth—study of the novel collision cone approach as a viable collision detection and avoidance tool in a 2-D dynamic environment. To allow this approach to be used in a practical situation we need to examine how the collision cone concept, and the avoidance strategies arising out of it, can be extended to a realistic situation where the robot tries to circumvent several moving obstacles while attempting to reach a specified stationary or moving goal point.

\section{REFERENCES}

[1] B. Aronov, S. Fortune, and G. Wilfong, "Minimum speed motions," Int. J. Robot. Res., vol. 10, pp. 228-239, June 1991.

[2] B. Barshan and H. F. Durrant-Whyte, "Inertial navigation systems for mobile robots," IEEE Trans. Robot. Automat., vol. 11, pp. 328-342, June 1995.

[3] B. Bhanu, O. Fagueras, B. Sridhar, and C. Thorpe, "Introduction to the special section on perception-based real-world navigation," IEEE Trans. Robot. Automat., vol. 10, pp. 725-727, Dec. 1994.

[4] A. Chakravarthy and D. Ghose, "Collision detection and avoidance: A collision cone approach," Tech. Rep. AE/GI/05-96, Dept. Aerospace Eng., Indian Inst. Sci., Bangalore, India, June 1996.

[5] B. Chazelle, "Approximation and decomposition of shapes," in $\mathrm{Ad}$ vances in Robotics Vol I: Algorithmic and Geometric Aspects of Robotics, J. T. Schwartz and C. K. Yap, Eds. Hillsdale, NJ: Lawrence Erlbaum, 1987, pp. 145-185.

[6] K. Fujimura, Motion Planning in Dynamic Environments. Tokyo, Japan: Springer-Verlag, 1991.

[7] _ "Motion planning amid transient obstacles," Int. J. Robot. Res., vol. 13, pp. 395-407, Oct. 1994.

[8] Y. K. Hwang and N. Ahuja, "Gross motion planning-A survey," ACM Comput. Surv., vol. 24, pp. 219-291, Sept. 1992.

[9] V. Hayward et al., "Efficient collision prediction among many moving obstacles," Int. J. Robot. Res., vol. 14, pp. 129-143, Apr. 1995.

[10] K. Kant and S. Zucker, "Toward efficient trajectory planning: The pathvelocity decomposition," Int. J. Robot. Res., vol. 5, no. 6, pp. 72-89, 1986.

[11] J. G. de Lamadrid, "Avoidance of obstacles with unknown trajectories: Locally optimal paths and periodic sensor readings," Int. J. Robot. Res., vol. 13, pp. 496-507, Dec. 1994.

[12] J. G. de Lamadrid and M. L. Gini, "Path tracking through uncharted moving obstacles," IEEE Trans. Syst., Man, Cybern., vol. 20, pp. 1408-1422, Nov./Dec. 1990.

[13] J.-C. Latombe, Robot Motion Planning. Boston, MA: Kluwer, 1991.
[14] C.-F. Lin, Modern Navigation, Guidance, and Control Processing. Englewood Cliffs, NJ: Prentice-Hall, 1991

[15] A. S. Locke, Guidance. Princeton, NJ: Van Nostrand, 1955.

[16] T. Lozano-Perez, "Spatial planning: A configuration space approach," IEEE Trans. Comput., vol. C-32, pp. 108-120, Feb. 1983.

[17] D. G. Luenberger, Linear and Nonlinear Programming. Reading, MA: Addison-Wesley, 1984

[18] H. Noborio, T. Naniwa, and S. Arimoto, "A quad-tree based path planning algorithm for a mobile robot," J. Robot. Syst., vol. 7, pp. 555-574, July 1990.

[19] M. Papageorgiou and T. Bauschert, "Stochastic optimal control of moving vehicles in a dynamic environment," Int. J. Robot. Res., vol. 13, pp. 343-354, Aug. 1994.

[20] H. L. Pastrick, S. M. Seltzer, and M. E. Warren, "Guidance laws for short-range tactical missiles," J. Guid. Contr., vol. 4, no. 2, pp. 98-108, Mar./Apr. 1981.

[21] J. Reif and M. Sharir, "Motion planning in the presence of moving obstacles," J. Assoc. Comput. Mach., vol. 41, pp. 764-790, July 1994.

[22] B. Roberts and B. Bhanu, "Inertial navigation sensor integrated motion analysis for autonomous vehicle navigation," J. Robot. Syst., vol. 9, pp. 817-842, Nov. 1992

[23] J. T. Schwartz and M. Sharir, "A survey of motion planning and related geometric algorithms," Artif. Intell., vol. 37, no. 1-3, pp. 157-169, Dec. 1988 .

[24] J. T. Schwartz and C. K. Yap, Eds., Advances in Robotics Vol I: Algorithmic and Geometric Aspects of Robotics. Hillsdale, NJ: Lawrence Erlbaum, 1987.

[25] R. Spence and S. Hutchinson, "An integrated architecture for robot motion planning and control in the presence of obstacles with unknown trajectories," IEEE Trans. Syst., Man, Cybern., vol. 25, pp. 100-110, Jan. 1995.

[26] J. P. H. Steele, "An algorithm with real-time response for avoiding moving obstacles," in Proc. IEEE Conf. Systems, Man, Cybernetics, 1989, pp. 551-556

[27] L. Tychonievich et al., "A maneuvering-board approach to path planning with moving obstacles," in Proc. 11th Int. Joint Conf. Artificial Intelligence, 1989 , pp. 1017-1021.

[28] L.-L. Wang and W.-H. Tsai, "Collision avoidance by a modified leastmean-square-error classification scheme for indoor autonomous land vehicle navigation," J. Robot. Syst., vol. 8, pp. 677-698, Sept. 1991.

[29] A. Zelinsky, "Mobile robot map making using sonar," J. Robot. Syst., vol. 8, pp. 557-577, Sept. 1991

[30] P. Zarchan, Tactical and Strategic Missile Guidance. Washington, DC: AIAA, 1990.

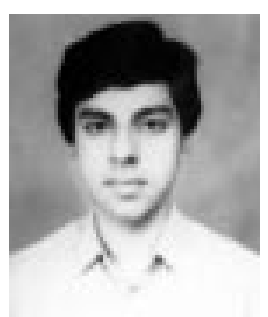

Animesh Chakravarthy received the Bachelor's degree in mechanical engineering in 1990 from Bangalore University, Bangalore, India, and the Master's degree in aerospace engineering in 1994 from the Indian Institute of Science, Bangalore.

$\mathrm{He}$ is presently a Scientist in the Flight Mechanics and Simulation Division, Aeronautical Development Agency, Bangalore. Apart from guidance and control theory, he has active interest in rigidbody/flexible structure dynamics, control of flexible structures, and robotics.

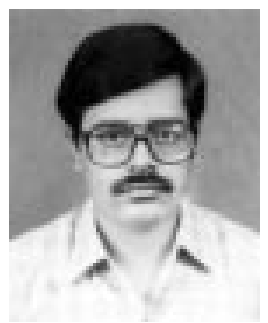

Debasish Ghose received the B.Sc.(Engg.) degree in electrical engineering from the Regional Engineering College, Rourkela, India, in 1982, and the M.E. and Ph.D. degrees, also in electrical engineering, from the Indian Institute of Science, Bangalore, in 1984 and 1990, respectively.

From 1984 to 1987, he was a Scientific Officer in the Joint Advanced Technology Programme, Indian Institute of Science, Bangalore, where he is presently an Associate Professor in the Department of Aerospace Engineering. His research interests are in the areas of guidance of aerospace vehicles, dynamic game theory, distributed computing, and robotics. He is a coauthor of the recent book Scheduling Divisible Loads in Parallel and Distributed Systems (Los Alamitos, CA: IEEE Computer Society Press). 